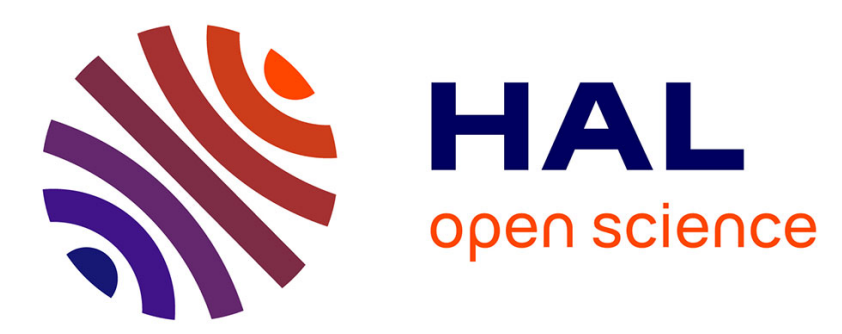

\title{
Consistent thermodynamic modelling of wire-reinforced geomaterials
}

\author{
Romain Laniel, Pierre Alart, Stéphane Pagano
}

\section{To cite this version:}

Romain Laniel, Pierre Alart, Stéphane Pagano. Consistent thermodynamic modelling of wirereinforced geomaterials. European Journal of Mechanics - A/Solids, 2007, 26 (5), pp.854-871. 10.1016/j.euromechsol.2007.01.003 . hal-00724461

\section{HAL Id: hal-00724461 https://hal.science/hal-00724461}

Submitted on 21 Aug 2012

HAL is a multi-disciplinary open access archive for the deposit and dissemination of scientific research documents, whether they are published or not. The documents may come from teaching and research institutions in France or abroad, or from public or private research centers.
L'archive ouverte pluridisciplinaire HAL, est destinée au dépôt et à la diffusion de documents scientifiques de niveau recherche, publiés ou non, émanant des établissements d'enseignement et de recherche français ou étrangers, des laboratoires publics ou privés. 


\title{
Consistent thermodynamic modelling of wire-reinforced geomaterials
}

\author{
R. Laniel ${ }^{\text {a,*}}{ }^{*}$ P. Alart $^{\text {a }}$, S. Pagano ${ }^{\text {a }}$ \\ ${ }^{\mathrm{a}}$ LMGC, UMR CNRS 5508, Université Montpellier II, CC 048 Place Eugène \\ Bataillon, 34095 Montpellier cedex 5, France
}

\begin{abstract}
The TexSol is a composite geomaterial: a sand matrix with a wire network reinforcement. For small strains a thermodynamic continuous model of the TexSol including the unilaterality of the wire network is postulated. This model is described by two potentials which depend on some internal variables and a state variable either strain or stress tensor (the choice of this last one gives two different ways of identification). The TexSol continuous model is implemented in a finite element code to retrieve the mechanical behavior given by discrete element numerical experiments.
\end{abstract}

Key words: geomaterial, unilaterality, thermodynamics

\section{Motivations}

\subsection{What is the TexSol?}

The civil-engineering constructions need plane stable floor. The environment configuration often forces civil engineers to setup huge embankments. Moreover, it can be interesting to reinforce them to ensure a better embankment mechanical behavior. A lot of different solutions can be used to reinforce soil but, in this paper, we focus our attention on the TexSol method.

The TexSol, created in 1984 by Leflaive Khay and Blivet from LCPC (Laboratoire Central des Ponts et Chaussées) (Leflaive and al., 1985), is a heterogeneous material obtained by mixing sand and wire network. This reinforced

\footnotetext{
* Corresponding author. Phone: +3346714 4537

Email address: romain.laniel@lmgc.univ-montp2.fr (R. Laniel).
}

Preprint submitted to Elsevier Science 24 January 2007 
material has a higher strength than sand without wire. Of course, the TexSol behavior depends on sand and wire parameters and its friction angle can be larger than sand by $0^{\circ}$ to $10^{\circ}$ (Khay and Gigan, 1990). The wire is described by its linear density in dtex units $\left(1 \mathrm{dtex}=10^{-7} \mathrm{~kg} \cdot \mathrm{m}^{-1}\right)$, ponderal content and stiffness. Classically, the wire density in a TexSol sample ranges between $10^{5} \mathrm{~m}^{-2}$ and $2.10^{5} \mathrm{~m}^{-2}$.

To make a TexSol bank, a machine named "Texsoleuse" is used. It proceeds by throwing sand and, at the same time, injecting wire. The wire is placed on the free surface of sand with random orientation. This machine carries out several passes to setup the bank. Figure 1 represents the TexSol microstructure. In the literature, we find two different continuous modellings. The model
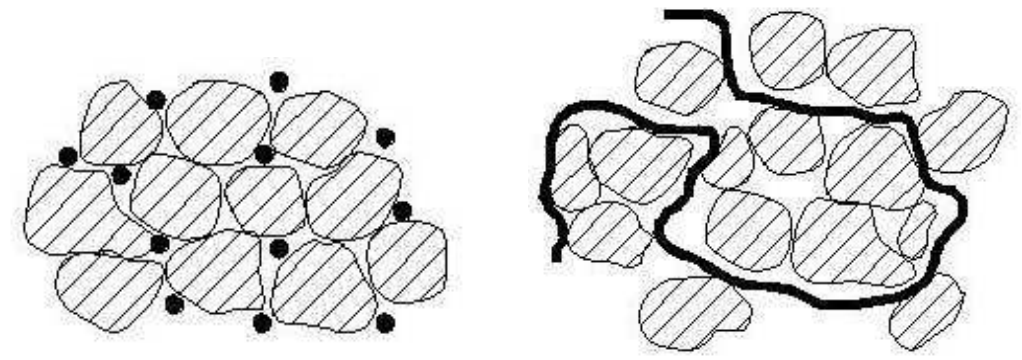

Fig. 1. Schematic TexSol sections

suggested in (Fremond, 2002) is non local and includes remote interactions (corresponding to the wire effects) but requires identification of their parameters using macroscopic experiments. Villard proposes a simpler local model in (Villard, 1988), (Villard and Jouve, 1989). It couples a standard model of sand and an equivalent unilateral elastic stiffness contribution corresponding to the wire network. This last contribution is activated only on the tension directions because of the unilateral behavior of wire. Our main work is to clearly define thermo-dynamical potentials of the Villard local model with both stress and strain formulations to identify the best-adapted one. Such a stage is useful before carrying out a homogenization procedure applied to an untypical material. In the absence of physical experiments, the identification of macroscopic model will be performed using numerical experiments. In the paper we use the continuum mechanics conventions.

\subsection{Assumptions of the continuous local model}

To couple the elasto-plastic model of the sand and the unilateral elastic model of the wire network, we have to consider some mechanical assumptions, which may be backed by numerical experiments performed with a discrete elements software (Dubois and Jean, 2003), (Moreau, 1999). We limit this study to the small strain framework; the stress tensor $\boldsymbol{\sigma}$ becomes a Cauchy stress tensor. 


\subsubsection{Stress additivity assumption}

In this paper, the stress additivity assumption of sand and wire network is assumed. Then, we write

$$
\boldsymbol{\sigma}=\boldsymbol{\sigma}_{s}+\boldsymbol{\sigma}_{w}
$$

where $\boldsymbol{\sigma}, \boldsymbol{\sigma}_{s}$ and $\boldsymbol{\sigma}_{w}$ are the second order stress tensors of the TexSol, sand and wire network, respectively.

This assumption seems to be consistent with the TexSol quasi-static behavior. We can get a good approximation of the stress tensor in numerical simulation of 2D granular matter (Mouraille, 2004) using the Weber stress tensor (Cambou and Jean, 2001),

$$
\boldsymbol{\sigma}_{\Omega}=\frac{1}{V_{\Omega}} \sum_{\alpha \in \Omega} l^{\alpha} \otimes r^{\alpha} .
$$

This tensor is an average over a domain $\Omega$ of volume $V_{\Omega}$, where $l^{\alpha}$ and $r^{\alpha}$ are the inter-center vector and the contact force vector of a contact $\alpha$. It may be asymmetric if inertial effects are not negligeable. For quasi-static processes this discrete tensor is a good candidate to represent a continuous stress tensor. Moreover, we can define such a tensor grain by grain with the approach of Moreau (Moreau, 1999). In this way, a wire network stress and a sand stress may be computed, to retrieve by addition the full TexSol stress (in the discrete simulation, the wire is modeled by a chain of beads with unilateral interactions (Laniel and al., 2005)). In a biaxial test (deviatoric load on a confined material) computed with LMGC90 using the Non Smooth Contact Dynamics method (Dubois and Jean, 2003), (Jean, 1999), we check the symmetry of the stress tensor even at large strains as long as the process remains slow (no inertial effects).

In the figure 2, we display the principal values of the Weber stress tensor (EV1,EV2), where the contributions of each components can be compared. The wire network is in tension and sand is in compression in both principal directions. These features may be also observed in the force network of granular samples.

\subsubsection{Non sliding assumption}

This second assumption is not as evident as the previous one. Although micro slidings occur between sand grains and wire, we assume that at the macroscopic level of the continuum model, the sand network does not slip with respect to the wire network. This assumption can be written by the equality 


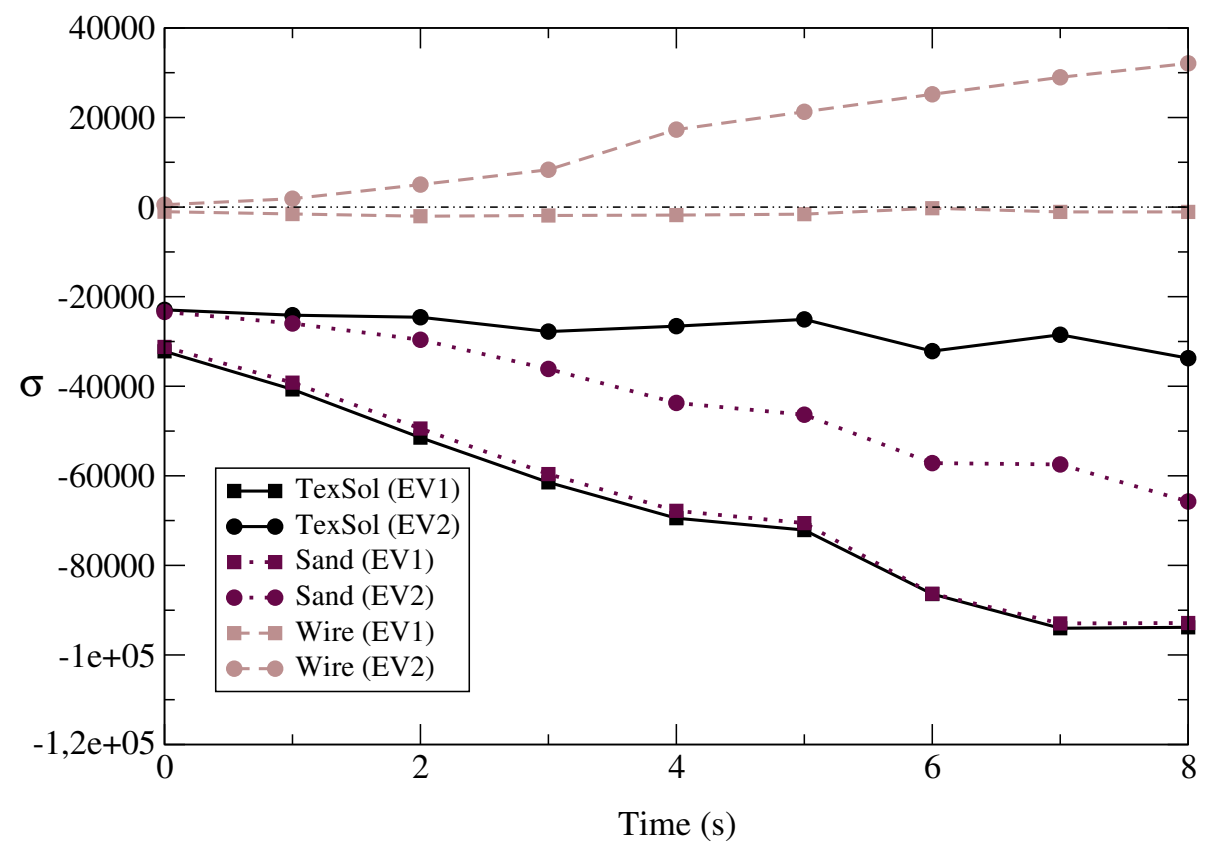

Fig. 2. Stress eigen values evolution in the TexSol

of the three strain rates,

$$
\dot{\varepsilon}_{s}=\dot{\varepsilon}_{w}=\dot{\varepsilon}
$$

where $\varepsilon_{s}, \varepsilon_{w}$ and $\varepsilon$ are the second order strain tensor of the sand, wire network and TexSol, respectively.

We have to be very careful with such a condition and define some validity domains for it. Indeed, the limits of this assumption are difficult to quantify and we will restrict the validation of the following continuum model to small strains.

\subsection{Role of the wire unilaterality}

The wire network contributes to the tensile stiffness of the composite material but not to the compression stifness (cf. figure 2). To model such a behavior at the macroscopic scale, it is convenient to introduce a unilateral condition in the behavior law of the wire network. This unilaterality accounts for two microscopic phenomena. The first one is the lack of bending strength of the

wire network viewed as a piece of cotton. The second one is the local buckling 
of short segments. The first aspect is not explicitly taken into account by a unilateral condition at the microscopic scale in our discrete numerical simulation since the chain of beads has no bending strength. The second aspect may be enforced bv introducing a unilateral interaction law between two successive beads

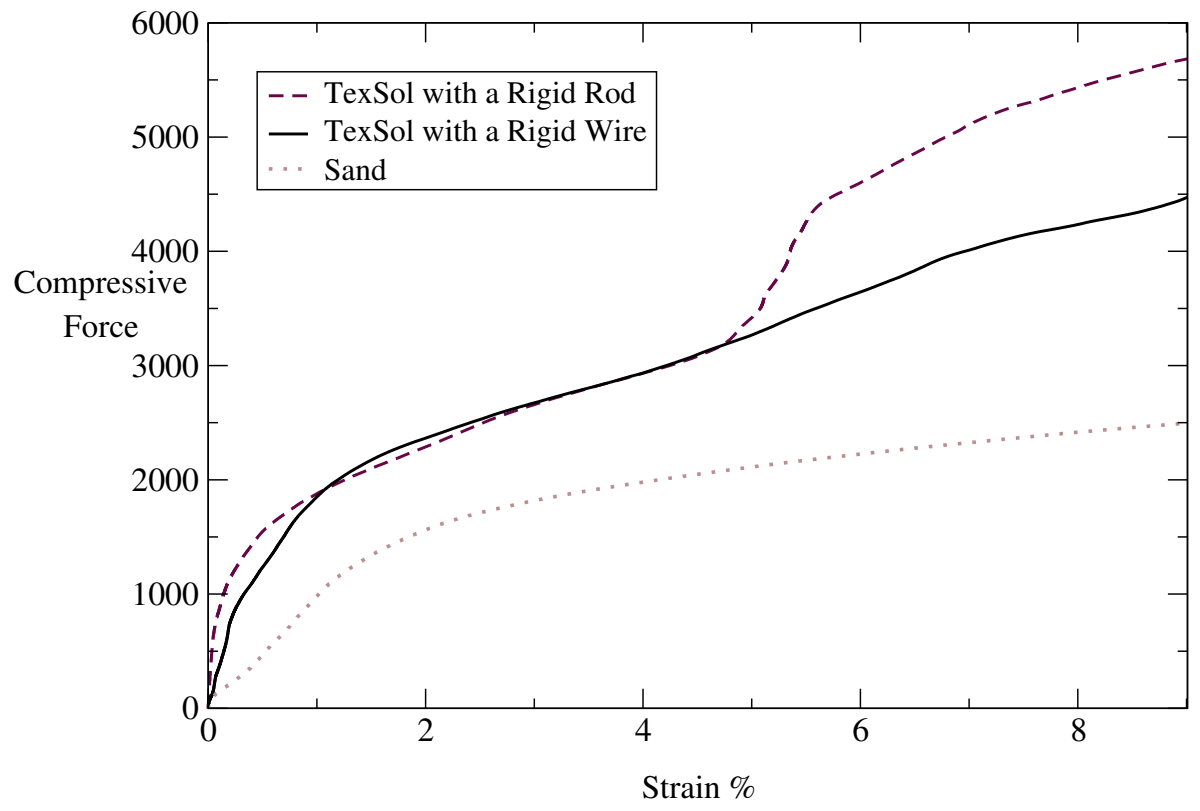

Fig. 3. Vertical force vs. vertical strain response with different models.

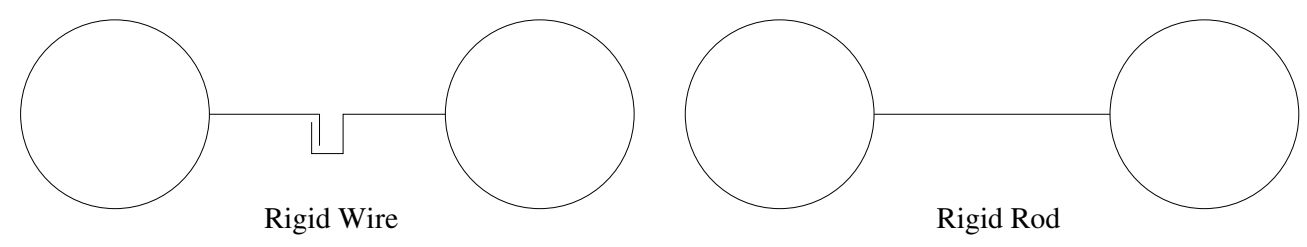

Fig. 4. Schematic discrete laws

denote by "rigid wire"; otherwise, we will refer to it as "rigid rod" (cf. figure 4) for bilateral law between beads. Figure 3 illustrates the difference of global behavior between the two simulations for biaxial test using the $L M G C 90$ software. Until $6 \%$ of deformation, the responses are almost identical, for larger deformations the "rigid rod" model leads to a rough increase of stiffness due to the appearance of compressive columns in the wire. Such a phenomenon does not seem very realistic and stems probably from a scale effect since the numerical sample is not fully representative of the material. In particular, the model of wire as a chain of beads generates unrealistic jamming effect with sand grains. 


\subsection{Why strain and stress formulations?}

In this paper, we propose to carry out a thermodynamic study with both strain and stress formulations. The interest of this work is in the identification possibilities of potentials parameters. Indeed, an experimenter performing tests on a sample has only access to the global strain. Our numerical investigations allow us to have access to finer data such as the local stress field throughout the sample. Moreover, the global stress tensor over the sample can be deduced by averaging.

The post-processing of numerical experiments mentioned in $\S 1.2 .1$ provides precise information on the stress fields in sand and wire network. The stress "unilaterality" in the wire is clearly established in figure 2. This observation could lead us to favour a stress formulation. But the finite element softwares are essentially developed using a strain formulation. Moreover, the unilaterality may be postulated either on the strain or on the stress according to the chosen approach. But these models are not dual of one another as we will see in the next section. Consequently, we propose in the following study strain formulations which are easily implementable. Dual stress formulations are provided when they can be analytically deduced by the Legendre - Fenchel transformation.

\section{$2 \quad$ A general thermodynamic framework}

In this part, we define potentials written with different state variables. These potentials have to satisfy the Clausius-Duhem inequality to be thermodynamically admissible. The notation conventions for an unspecified variable are $X$ for a scalar or a vector, $\boldsymbol{X}$ for a second or third order tensor and $\mathbb{X}$ for a fourth or higher order tensor.

\subsection{Strain versus stress approach in thermodynamics}

This work must be as exhaustive as possible, when passing from unspecified state variables to its dual. We thus use the Legendre-Fenchel transformation (Moreau, 1966), to carry out our study with both strain and stress formulations. Let us write the Clausius-Duhem inequality where $u$ is the internal energy, $s$ the massic entropy, $q$ the heat flow vector and $T$ the temperature,

$$
\boldsymbol{\sigma}: \dot{\varepsilon}-\rho(\dot{u}-T \dot{s})-\frac{1}{T} q \cdot \nabla T \geq 0
$$


where $\nabla$ is the gradient operator. The intrinsic dissipation depends on a state variable $\boldsymbol{X}$ (or its dual $\boldsymbol{X}^{*}$ ), some internal variables $\alpha=\left\{\alpha_{1}, \cdots, \alpha_{p}\right\}$ (each internal variable can be scalar, vectorial or tensorial) and the temperature $T$. It can also be expressed with the free energy $\psi$ or its Legendre-Fenchel transformation $\psi^{*}$ with respect to the state variable $\boldsymbol{X}$,

$$
\text { and } \begin{aligned}
\psi(\boldsymbol{X}, \alpha, T) & =u(\boldsymbol{X}, \alpha, T)-T s(\boldsymbol{X}, \alpha, T) \\
\psi^{*}\left(\boldsymbol{X}^{*}, \alpha, T\right) & =\sup _{\overline{\boldsymbol{X}}}\left\{\overline{\boldsymbol{X}}: \boldsymbol{X}^{*}-\psi(\overline{\boldsymbol{X}}, \alpha, T)\right\} \\
& =\boldsymbol{X}: \boldsymbol{X}^{*}-\psi(\boldsymbol{X}, \alpha, T)
\end{aligned}
$$

where $\boldsymbol{X}$ is the argument of the supremum. Considering either $\boldsymbol{X}$ or $\boldsymbol{X}^{*}$, we find two expressions of the Clausius-Duhem inequality,

$$
\begin{array}{r}
\boldsymbol{\sigma}: \dot{\varepsilon}-\rho\left[\frac{\partial \psi}{\partial \boldsymbol{X}}: \dot{\boldsymbol{X}}+\left(s+\frac{\partial \psi}{\partial T}\right) \dot{T}+\frac{\partial \psi}{\partial \alpha_{m}} \dot{\alpha}_{m}\right]-\frac{q}{T} \cdot \nabla T \geq 0 \\
\boldsymbol{\sigma}: \dot{\varepsilon}-\rho\left[\dot{\boldsymbol{X}}: \boldsymbol{X}^{*}+\left(\boldsymbol{X}-\frac{\partial \psi^{*}}{\partial \boldsymbol{X}^{*}}\right): \dot{\boldsymbol{X}}^{*}+\left(s-\frac{\partial \psi^{*}}{\partial T}\right) \dot{T}-\frac{\partial \psi^{*}}{\partial \alpha_{m}} \dot{\alpha}_{m}\right] \\
-\frac{q}{T} \cdot \nabla T \geq 0
\end{array}
$$

Using the Helmholtz postulate (which can be applied with the generalized standard materials assumption (Halphen and Nguyen, 1975)) and the previous definitions, we are now able to deduce the state laws,

$$
\begin{array}{cc}
\text { Primal state laws } & \text { Dual state laws } \\
\boldsymbol{X}^{*} \in \partial_{\boldsymbol{X}} \psi(\boldsymbol{X}, \alpha, T) & \boldsymbol{X} \in \partial_{\boldsymbol{X}^{*} \psi^{*}}\left(\boldsymbol{X}^{*}, \alpha, T\right) \\
-s \in \partial_{T} \psi(\boldsymbol{X}, \alpha, T) & s \in \partial_{T} \psi^{*}\left(\boldsymbol{X}^{*}, \alpha, T\right) \\
-\frac{1}{\rho} A_{m} \in \partial_{\alpha_{m}} \psi(\boldsymbol{X}, \alpha, T) & \frac{1}{\rho} A_{m} \in \partial_{\alpha_{m}} \psi^{*}\left(\boldsymbol{X}^{*}, \alpha, T\right),
\end{array}
$$

where $A_{m}$ is the thermodynamic force associated with $\alpha_{m}$. Formally, we use subdifferentials instead of derivatives; for a function $f$ at the point $p$ it is defined by $\partial_{p} f=\{q \mid \forall r, f(r)-f(p) \geq q .(r-p)\}$. If convexity is not required, previous relations still hold using the Clarke subdifferential (Clarke, 1983). Then, the primal and dual forms are not necessarily equivalent. In the general case, the Clausius-Duhem inequality (4) or (5) can be reduced to a dot product 
of a vector flow and a vector force,

$$
\underbrace{\left[\begin{array}{c}
\hat{\boldsymbol{\sigma}} \\
A \\
\nabla T
\end{array}\right]^{T}}_{\text {force }} \cdot \underbrace{\left[\begin{array}{c}
\dot{\widehat{\boldsymbol{\varepsilon}}} \\
\dot{\alpha} \\
-\frac{q}{T}
\end{array}\right]}_{\text {flow }} \geq 0 \quad \text { where }\left\{\begin{array}{l}
\dot{\widehat{\boldsymbol{\varepsilon}}}=\dot{\boldsymbol{\varepsilon}} \text { or } \dot{\boldsymbol{\varepsilon}}^{i r} \\
\hat{\boldsymbol{\sigma}}=\boldsymbol{\sigma}^{i r} \text { or } \boldsymbol{\sigma}
\end{array}\right.
$$

The flow variables have to be related through evolution laws to the force variables. To satisfy the inequality (7) some assumptions may be added to these relations. It is convenient to introduce a dissipation potential $\varphi$ from which the evolution laws are derived. By duality, a force function $\varphi^{*}$ is automatically defined using the Legendre-Fenchel transformation,

Primal complementary laws Dual complementary laws

$$
\begin{array}{cc}
\hat{\boldsymbol{\sigma}} \in \partial_{\hat{\boldsymbol{\varepsilon}}} \varphi\left(\dot{\widehat{\boldsymbol{\varepsilon}}}, \dot{\alpha},-\frac{q}{T}\right) & \dot{\widehat{\boldsymbol{\varepsilon}}} \in \partial_{\widehat{\boldsymbol{\sigma}}} \varphi^{*}\left(\widehat{\boldsymbol{\sigma}}, \dot{\alpha},-\frac{q}{T}\right) \\
A \in \partial_{\dot{\alpha}} \varphi\left(\dot{\widehat{\boldsymbol{\varepsilon}}}, \dot{\alpha},-\frac{q}{T}\right) & -A \in \partial_{\dot{\alpha}} \varphi^{*}\left(\widehat{\boldsymbol{\sigma}}, \dot{\alpha},-\frac{q}{T}\right) \\
\nabla T \in \partial_{\left(-\frac{q}{T}\right)} \varphi\left(\dot{\widehat{\boldsymbol{\varepsilon}}}, \dot{\alpha},-\frac{q}{T}\right) & -\nabla T \in \partial_{\left(-\frac{q}{T}\right)} \varphi^{*}\left(\widehat{\boldsymbol{\sigma}}, \dot{\alpha},-\frac{q}{T}\right) .
\end{array}
$$

To satisfy the Clausius-Duhem inequality, some assumptions on the dissipation potential are necessary. For simplicity, we consider now an isothermal process. The left-hand side of the inequality is reduced to,

$$
\widehat{\boldsymbol{\sigma}}: \dot{\widehat{\boldsymbol{\varepsilon}}}+A \dot{\alpha}=\frac{\partial \varphi}{\partial \dot{\widehat{\boldsymbol{\varepsilon}}}}: \dot{\widehat{\boldsymbol{\varepsilon}}}+\frac{\partial \varphi}{\partial \dot{\alpha}} \dot{\alpha}=\partial \varphi(\dot{\widehat{\boldsymbol{\varepsilon}}}, \dot{\alpha}) \cdot(\dot{\widehat{\boldsymbol{\varepsilon}}}, \dot{\alpha})
$$

and the primal state laws are summarized as $(\widehat{\boldsymbol{\sigma}}, A) \in \partial \varphi(\dot{\widehat{\boldsymbol{\varepsilon}}}, \dot{\alpha})$, the potential $\varphi$ being a convex function. With a convex analysis characterisation of the subdifferential we write

$$
\forall(\boldsymbol{x}, y) \quad \varphi(\dot{\widehat{\boldsymbol{\varepsilon}}}, \dot{\alpha})-\varphi(\boldsymbol{x}, y) \leq((\dot{\widehat{\boldsymbol{\varepsilon}}}, \dot{\alpha})-(\boldsymbol{x}, y)) \cdot(\hat{\boldsymbol{\sigma}}, A)
$$

Moreover, if $\varphi$ is minimum in $(\mathbf{0}, 0)$, the Clausius-Duhem inequality is then satisfied (Suquet, 1982),

$$
\widehat{\boldsymbol{\sigma}}: \dot{\widehat{\boldsymbol{\varepsilon}}}+A \dot{\alpha} \geq \varphi(\dot{\widehat{\boldsymbol{\varepsilon}}}, \dot{\alpha})-\varphi(\mathbf{0}, 0) \geq 0
$$

Similar properties are required for $\varphi^{*}$ to recover the Clausius-Duhem inequality. Generally, we distinguish the reversible and irreversible parts of the transformation. We thus postulate an additive decomposition for both reversible 
and irreversible parts of the strain tensor $\varepsilon=\varepsilon^{r}+\varepsilon^{i r}$ (which is classical for the so called small strain assumption) and the stress tensor $\boldsymbol{\sigma}=\boldsymbol{\sigma}^{r}+\boldsymbol{\sigma}^{i r}$. The reversible / irreversible splitting of $\boldsymbol{\sigma}$ is less classical. To illustrate its interest, remark that possible residual stresses may be accounted for in the irreversible part.

At this stage, we have to choose the external state variable $\boldsymbol{X}$ for the strain formulation and consequently $\boldsymbol{X}^{*}$ for the stress formulation. It is usual to consider for $\boldsymbol{X}$ the total strain tensor $\boldsymbol{\varepsilon}$. By the way, the reversible stress $\boldsymbol{\sigma}^{r}$ appears in the state law and becomes the state variable in the dual stress formulation. But we can also use the reversible strain part $\varepsilon^{r}$ and deduce the full stress tensor $\boldsymbol{\sigma}$ as the dual state variable (cf. table I). The first column

\begin{tabular}{|c|c|c|c|}
\hline \multicolumn{2}{|c|}{$\begin{array}{l}\text { State variable: } \boldsymbol{\varepsilon}^{r} \\
\qquad \boldsymbol{\sigma}: \varepsilon^{i r}+A_{m} \dot{\alpha}_{m}-\frac{q}{T} \nabla T \geq 0\end{array}$} & \multicolumn{2}{|c|}{$\begin{array}{l}\text { State variable: } \boldsymbol{\sigma} \\
\qquad \dot{\varepsilon}^{\text {ir }}: \boldsymbol{\sigma}+A_{m} \dot{\alpha}_{m}-\frac{q}{T} \nabla T \geq 0\end{array}$} \\
\hline free energy: $\psi$ & $\begin{array}{l}\text { Dissipation potential: } \\
\varphi\end{array}$ & Free enthalpy: $\psi^{*}$ & Force function: $\varphi^{*}$ \\
\hline $\begin{array}{c}\frac{1}{\rho} \boldsymbol{\sigma} \in \partial_{\boldsymbol{\varepsilon}^{r}} \psi \\
-s \in \partial_{T} \psi \\
-\frac{1}{\rho} A_{m} \in \partial_{\alpha_{m}} \psi\end{array}$ & $\begin{array}{c}\boldsymbol{\sigma} \in \partial_{\dot{\varepsilon}}{ }_{i r} \varphi \\
A_{m} \in \partial_{\dot{\alpha}_{m}} \varphi \\
\nabla T \in \partial_{\left(-\frac{q}{T}\right)} \varphi \\
\end{array}$ & $\begin{array}{c}\frac{1}{\rho} \varepsilon^{r} \in \partial_{\boldsymbol{\sigma}} \psi^{*} \\
s \in \partial_{T} \psi^{*} \\
\frac{1}{\rho} A_{m} \in \partial_{\alpha_{m}} \psi^{*}\end{array}$ & $\begin{array}{c}\dot{\boldsymbol{\varepsilon}}^{\imath r} \in \partial_{\sigma} \varphi^{*} \\
-A_{m} \in \partial_{\alpha_{m}} \varphi^{*} \\
-\nabla T \in \partial_{\left(-\frac{q}{T}\right)} \varphi^{*}\end{array}$ \\
\hline \multicolumn{2}{|c|}{$\begin{array}{l}\text { State variable: } \boldsymbol{\varepsilon} \\
\qquad \boldsymbol{\sigma}^{i r}: \dot{\varepsilon}+A_{m} \dot{\alpha}_{m}-\frac{q}{T} \nabla T \geq 0\end{array}$} & \multicolumn{2}{|c|}{$\begin{array}{l}\text { State variable: } \boldsymbol{\sigma}^{r} \\
\qquad \dot{\boldsymbol{\varepsilon}}: \boldsymbol{\sigma}^{i r}+A_{m} \dot{\alpha}_{m}-\frac{q}{T} \nabla T \geq 0\end{array}$} \\
\hline Free energy: $\psi$ & $\begin{array}{l}\text { Dissipation potential: } \\
\varphi\end{array}$ & Free enthalpy: $\psi^{*}$ & Force function: $\varphi^{*}$ \\
\hline $\begin{array}{c}\frac{1}{\rho} \boldsymbol{\sigma}^{r} \in \partial_{\varepsilon} \psi \\
-s \in \partial_{T} \psi \\
-\frac{1}{\rho} A_{m} \in \partial_{\alpha_{m}} \psi\end{array}$ & $\begin{array}{c}\sigma^{i r} \in \partial_{\dot{\varepsilon}} \varphi \\
A_{m} \in \partial_{\alpha_{m}} \varphi \\
\nabla T \in \partial_{\left(-\frac{q}{T}\right)} \varphi \\
\end{array}$ & $\begin{array}{c}\frac{1}{\rho} \varepsilon \in \partial_{\boldsymbol{\sigma}^{r}} \psi^{*} \\
s \in \partial_{T} \psi^{*} \\
\frac{1}{\rho} A_{m} \in \partial_{\alpha_{m}} \psi^{*} \\
\end{array}$ & $\begin{array}{c}\dot{\boldsymbol{\varepsilon}} \in \partial_{\boldsymbol{\sigma}^{i r}} \varphi^{*} \\
-A_{m} \in \partial_{\alpha_{m}} \varphi^{*} \\
-\nabla T \in \partial_{\left(-\frac{q}{T}\right)} \varphi^{*}\end{array}$ \\
\hline
\end{tabular}

Table I

Strain versus stress formulations

expresses the primal model using $\varepsilon^{r}$ or $\varepsilon$ as state variable. The second column provides the corresponding dual formulations.

\section{2 $1 D$ model of reinforced geomaterial}

Let us apply previous results to a rheological 1D model of TexSol taking into account the wire unilaterality.

\subsubsection{Strain formulation}

We choose to superpose a classical 1D model of elasto-plasticity with hardening for sand (Lemaitre and Chaboche, 1990) and a 1D unilateral model of 
elasticity for wire. We thus propose the two potentials $\psi$ (free energy) and $\varphi$ (dissipation potential) depending on the external state variable $\varepsilon$ and on the internal variable $\varepsilon_{2}$ as shown in the figure 5 ,

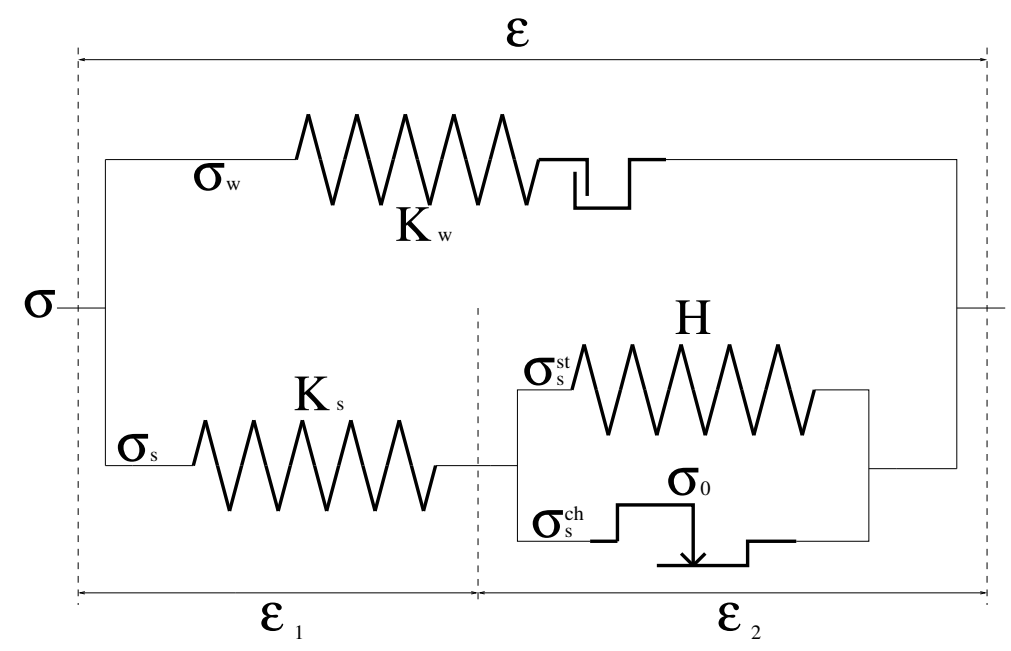

Fig. 5. Rheological TexSol diagram

$$
\begin{aligned}
& \psi\left(\varepsilon, \varepsilon_{2}\right)=\left\{\begin{array}{ll}
\psi_{1}\left(\varepsilon, \varepsilon_{2}\right) & \text { if } \varepsilon \in C_{1} \\
\psi_{2}\left(\varepsilon, \varepsilon_{2}\right) & \text { if } \varepsilon \in C_{2}
\end{array},\right. \\
& \varphi\left(\dot{\varepsilon}, \dot{\varepsilon}_{2}\right)=\sigma_{0}\left|\dot{\varepsilon}_{2}\right|
\end{aligned}
$$

where

$$
\begin{array}{ll}
\psi_{1}\left(\varepsilon, \varepsilon_{2}\right)=\frac{1}{2} K_{w} \varepsilon^{2}+\frac{1}{2} K_{s}\left(\varepsilon-\varepsilon_{2}\right)^{2}+\frac{1}{2} H \varepsilon_{2}{ }^{2} & C_{1}=\{\varepsilon \in \mathbb{R} \mid \varepsilon \geq 0\} \\
\psi_{2}\left(\varepsilon, \varepsilon_{2}\right)=\frac{1}{2} K_{s}\left(\varepsilon-\varepsilon_{2}\right)^{2}+\frac{1}{2} H \varepsilon_{2}{ }^{2} & C_{2}=\{\varepsilon \in \mathbb{R} \mid \varepsilon \leq 0\}
\end{array}
$$

and $\sigma_{0}$ the stress threshold. According to table I the state and complementary laws are derived,

State laws:

$$
\sigma^{r}=\frac{\partial \psi}{\partial \varepsilon}=K_{w}\langle\varepsilon\rangle+K_{s}\left(\varepsilon-\varepsilon_{2}\right), A=-\frac{\partial \psi}{\partial \varepsilon_{2}}=K_{s}\left(\varepsilon-\varepsilon_{2}\right)-H \varepsilon_{2}
$$

Complementary laws:

$$
\sigma^{i r}=\frac{\partial \varphi}{\partial \dot{\varepsilon}}=0, A \in \partial_{\dot{\varepsilon_{2}}} \varphi=\left\{\begin{array}{ll}
\left\{\operatorname{sgn}\left(\dot{\varepsilon_{2}}\right) \sigma_{0}\right\} & \text { if } \dot{\varepsilon_{2}} \in \mathbb{R}^{*} \\
{\left[-\sigma_{0}, \sigma_{0}\right]} & \text { if } \dot{\varepsilon_{2}}=0
\end{array},\right.
$$

where $\langle\varepsilon\rangle=\max (0, \varepsilon)$ is the non negative part of $\varepsilon$ and $\operatorname{sgn}($.$) is the signum$ operator. 


\subsubsection{Stress formulation}

To determine the stress formulation, we have to calculate the LegendreFenchel transformations of $\psi$ and $\varphi$ which are not always analytically accessible. However, we can use the following general result convenient for piecewise smooth functions.

Proposition 1 Consider a non overlapping splitting $\left(C_{i}\right)_{i=1, n}$ of the strain space $\mathbb{R}^{3 \times 3}, \cup_{i=1}^{n} C_{i}=\mathbb{R}^{3 \times 3}, C_{i}$ close convex cone with mes $\left(C_{i} \cap C_{j}\right)=0$, $i \neq j$. If $\psi(\varepsilon)$ is piecewise defined by $\psi(\varepsilon)=\psi_{i}(\varepsilon)$ if $\varepsilon \in C_{i}, i=1, \ldots, n$ then

$$
\psi^{*}(\boldsymbol{\sigma})=\sup _{i}\left\{\left(\psi_{i}^{*} \nabla \mathrm{I}_{C_{i}^{\circ}}\right)(\boldsymbol{\sigma})\right\}
$$

Proof: Let us recall the definition of inf-convolution of two functions $f$ and $g$ (Moreau, 1966), the indicator function of a convex set $A$ and the polar cone $C^{\circ}$ of $C$,

$$
\begin{array}{ll}
\circ & (f \nabla g)(\varepsilon)=\inf _{\varepsilon=\varepsilon_{1}+\varepsilon_{2}}\left\{f\left(\varepsilon_{1}\right)+f\left(\varepsilon_{2}\right)\right\} \\
\circ & \mathrm{I}_{A}(\varepsilon)=\left\{\begin{array}{cc}
0 & \text { if } \varepsilon \in A \\
+\infty & \text { if } \varepsilon \notin A
\end{array}\right. \\
\circ & C^{\circ}=\{\boldsymbol{\sigma} \mid \boldsymbol{\varepsilon}: \boldsymbol{\sigma} \leq 0, \forall \boldsymbol{\varepsilon} \in C\}
\end{array}
$$

According to classical rules of convex analysis,

$$
\begin{aligned}
\psi^{*}(\boldsymbol{\sigma}) & =\sup _{\overline{\boldsymbol{\varepsilon}}}\left\{\boldsymbol{\sigma}: \overline{\boldsymbol{\varepsilon}}-\inf _{i}\left\{\psi_{i}(\overline{\boldsymbol{\varepsilon}})+\mathrm{I}_{C_{i}}(\overline{\boldsymbol{\varepsilon}})\right\}\right\} \\
& =\sup _{i}\left\{\sup _{\overline{\boldsymbol{\varepsilon}}}\left\{\boldsymbol{\sigma}: \overline{\boldsymbol{\varepsilon}}-\psi_{i}(\overline{\boldsymbol{\varepsilon}})-\mathrm{I}_{C_{i}}(\overline{\boldsymbol{\varepsilon}})\right\}\right\} \\
& =\sup _{i}\left\{\left(\psi_{i}+\mathrm{I}_{C_{i}}\right)^{*}(\boldsymbol{\sigma})\right\} \\
& =\sup _{i}\left\{\left(\psi_{i}^{*} \nabla \mathrm{I}_{C_{i}^{\circ}}\right)(\boldsymbol{\sigma})\right\} .
\end{aligned}
$$

For the 1D model the split into two half spaces is obvious and the analytical forms of conjugate functions from (9) are accessible,

$$
\begin{aligned}
& \psi_{1}^{*}\left(\sigma^{r}, \varepsilon_{2}\right)=\frac{\left(\sigma^{r}+K_{s} \varepsilon_{2}\right)^{2}}{2\left(K_{w}+K_{s}\right)}-\frac{\left(K_{s}+H\right) \varepsilon_{2}^{2}}{2}, C_{1}^{\circ}=\{\sigma \in \mathbb{R} \mid \sigma \leq 0\} \\
& \psi_{2}^{*}\left(\sigma^{r}, \varepsilon_{2}\right)=\frac{\left(\sigma^{r}+K_{s} \varepsilon_{2}\right)^{2}}{2 K_{s}}-\frac{\left(K_{s}+H\right) \varepsilon_{2}^{2}}{2}, C_{2}^{\circ}=\{\sigma \in \mathbb{R} \mid \sigma \geq 0\}
\end{aligned}
$$


Using proposition 1, we obtain successively,

$$
\begin{aligned}
& \psi_{1}^{*} \nabla \mathrm{I}_{C_{1}^{\circ}}= \begin{cases}-\frac{\left(K_{s}+H\right) \varepsilon_{2}^{2}}{2} & \text { if } \sigma^{r}+K_{s} \varepsilon_{2} \leq 0 \\
\frac{\left(\sigma^{r}+K_{s} \varepsilon_{2}\right)^{2}}{2\left(K_{w}+K_{s}\right)}-\frac{\left(K_{s}+H\right) \varepsilon_{2}^{2}}{2} & \text { if } \sigma^{r}+K_{s} \varepsilon_{2} \geq 0\end{cases} \\
& \psi_{2}^{*} \nabla \mathrm{I}_{C_{2}^{\circ}}= \begin{cases}-\frac{\left(K_{s}+H\right) \varepsilon_{2}{ }^{2}}{2} & \text { if } \sigma^{r}+K_{s} \varepsilon_{2} \geq 0 \\
\frac{\left(\sigma^{r}+K_{s} \varepsilon_{2}\right)^{2}}{2 K_{s}}-\frac{\left(K_{s}+H\right) \varepsilon_{2}^{2}}{2} & \text { if } \sigma^{r}+K_{s} \varepsilon_{2} \leq 0 .\end{cases}
\end{aligned}
$$

Finally,

$$
\psi^{*}\left(\sigma^{r}, \varepsilon_{2}\right)=\left\{\begin{array}{ll}
\frac{\left(\sigma^{r}+K_{s} \varepsilon_{2}\right)^{2}}{2\left(K_{w}+K_{s}\right)}-\frac{\left(K_{s}+H\right) \varepsilon_{2}^{2}}{2} & \text { if } \sigma^{r}+K_{s} \varepsilon_{2} \geq 0 \\
\frac{\left(\sigma^{r}+K_{s} \varepsilon_{2}\right)^{2}}{2 K_{s}}-\frac{\left(K_{s}+H\right) \varepsilon_{2}{ }^{2}}{2} & \text { if } \sigma^{r}+K_{s} \varepsilon_{2} \leq 0
\end{array} .\right.
$$

The Legendre-Fenchel transformation of the dissipation potential is computed classically from (10),

$$
\varphi^{*}\left(\sigma^{i r}, \dot{\varepsilon_{2}}\right)=\mathrm{I}_{\{0\}}\left(\sigma^{i r}\right)-\sigma_{0}\left|\dot{\varepsilon_{2}}\right|
$$

From the equation (12) we implicitly get $\sigma^{i r}=0$. The state and complementary laws in the stress formulation are derived in a straightforward manner

State laws:

$$
\begin{aligned}
& \varepsilon=\frac{\partial \psi^{*}}{\partial \sigma^{r}}\left(\sigma^{i r}, \varepsilon_{2}\right)=\left\{\begin{array}{l}
\frac{\sigma^{r}+K_{s} \varepsilon_{2}}{K_{w}+K_{s}} \text { if } \sigma^{r}+K_{s} \varepsilon_{2} \geq 0 \\
\frac{\sigma^{r}+K_{s} \varepsilon_{2}}{K_{s}} \text { if } \sigma^{r}+K_{s} \varepsilon_{2} \leq 0
\end{array}\right. \\
& A=\frac{\partial \psi^{*}}{\partial \varepsilon_{2}}\left(\sigma^{i r}, \varepsilon_{2}\right)= \begin{cases}\frac{K_{s}}{K_{w}+K_{s}}\left(\sigma^{r}+K_{s} \varepsilon_{2}\right)-\left(K_{s}+H\right) \varepsilon_{2} & \text { if } \sigma^{r}+K_{s} \varepsilon_{2} \geq 0 \\
\sigma^{r}+K_{s} \varepsilon_{2}-\left(K_{s}+H\right) \varepsilon_{2} & \text { if } \sigma^{r}+K_{s} \varepsilon_{2} \leq 0\end{cases}
\end{aligned}
$$

Complementary laws:

$$
\dot{\varepsilon} \in \partial_{\sigma^{i r}} \varphi^{*}\left(\sigma^{i r}, \dot{\varepsilon}_{2}\right)=\mathbb{R}
$$

$$
A \in-\partial_{\dot{\varepsilon}_{2}} \varphi^{*}\left(\sigma^{i r}, \dot{\varepsilon_{2}}\right)=\left\{\begin{array}{lll}
\left\{\operatorname{sgn}\left(\dot{\varepsilon}_{2}\right) \sigma_{0}\right\} & \text { si } & \dot{\varepsilon_{2}} \in \mathbb{R}^{*} \\
{\left[-\sigma_{0}, \sigma_{0}\right]} & \text { si } & \dot{\varepsilon_{2}}=0
\end{array} .\right.
$$

This set of equations is equivalent to the one obtained with the strain formulation $\S 2.2 .1$. 


\section{Strain and stress approach for 3D models}

The three dimensional effects can not be neglected in modelling the complex microstructure of the TexSol material. To define a 3D model, we follow the previous 1D approach superposing a classical elastic plastic behavior for the sand and a unilateral elastic one for the wire network. Simple and sophisticated

unilaterality conditions may be considered leading to different formulations more or less easy to handle in a general primal / dual framework.

\subsection{D thermodynamical potentials of the sand}

First of all, let us recall that the stress tensor can be split into a spherical part and a deviatoric one,

$$
\boldsymbol{\sigma}=\underbrace{\frac{1}{3} \operatorname{tr}(\boldsymbol{\sigma}) \boldsymbol{I}}_{\text {spherical }}+\underbrace{\boldsymbol{S}}_{\text {deviatoric }}
$$

where $\boldsymbol{I}$ is the identity second order tensor $\left(I_{i j}=\delta_{i j}\right)$ and $\operatorname{tr}($.$) is the trace$ operator. Let us introduce the spherical projection tensor $\mathbb{S}=\frac{1}{3} \boldsymbol{I} \otimes \boldsymbol{I}$ and the deviatoric projection tensor $\mathbb{D}=\mathbb{I}-\mathbf{S}$ where $\mathbb{I}$ is the identity fourth order tensor $\left(I_{i j k l}=\frac{1}{2}\left(\delta_{i k} \delta_{j l}+\delta_{i l} \delta_{j k}\right)\right)$. In a classical model the state variable is sand's full strain $\varepsilon_{s}$, the internal variables contain the plastic strain $\varepsilon_{s}^{p}$, the kinematic and isotropic hardening variables $\boldsymbol{\alpha}$ and $p$ (Wood, 1990). The free energy $\psi_{s}$ has the following form,

$$
\psi_{s}\left(\boldsymbol{\varepsilon}_{s}, \boldsymbol{\varepsilon}_{s}^{p}, \boldsymbol{\alpha}, p\right)=\frac{1}{2}\left(\varepsilon_{s}-\varepsilon_{s}^{p}\right): \mathbb{K}_{s}\left(\boldsymbol{\varepsilon}_{s}-\boldsymbol{\varepsilon}_{s}^{p}\right)+\frac{H_{k}}{2} \boldsymbol{\alpha}: \boldsymbol{\alpha}+\frac{H_{i}}{2} p^{2} .
$$

where $\mathbb{K}_{s}, H_{k}$ and $H_{i}$ are stiffness coefficients. The state laws are directly derived from it,

$$
\begin{aligned}
\boldsymbol{\sigma}_{s}^{r} & =\frac{\partial \psi_{s}}{\partial \boldsymbol{\varepsilon}_{s}}=\mathbb{K}_{s}\left(\boldsymbol{\varepsilon}_{s}-\boldsymbol{\varepsilon}_{s}^{p}\right) \\
\boldsymbol{A} & =-\frac{\partial \psi_{s}}{\partial \boldsymbol{\varepsilon}_{s}^{p}}=\mathbb{K}_{s}\left(\boldsymbol{\varepsilon}_{s}-\boldsymbol{\varepsilon}_{s}^{p}\right) \\
\boldsymbol{\chi} & =-\frac{\partial \psi_{s}}{\partial \boldsymbol{\alpha}}=-H_{k} \boldsymbol{\alpha} \\
R & =-\frac{\partial \psi_{s}}{\partial p}=-H_{i} p .
\end{aligned}
$$

To derive the complementary laws, it is more convenient to define the force function $\varphi^{*}$ instead of the dissipation potential $\varphi$,

$$
\varphi_{s}^{*}\left(\boldsymbol{\sigma}_{s}^{i r}, \boldsymbol{A}, \boldsymbol{\chi}, R\right)=\mathrm{I}_{\{\mathbf{0}\}}\left(\boldsymbol{\sigma}_{s}^{i r}\right)+\mathrm{I}_{\Omega(\boldsymbol{\chi}, R)}(\boldsymbol{A})
$$


where $\Omega(\boldsymbol{\chi}, R)=\{\boldsymbol{A} \mid F(\boldsymbol{A}, \boldsymbol{\chi}, R) \leq 0\}$ is the elastic domain bounded by the Drucker - Prager criterion $F$ defined by (Drucker and Prager, 1952),

$$
F(\boldsymbol{A}, \boldsymbol{\chi}, R)=\sqrt{J_{2}(\boldsymbol{A}-\boldsymbol{\chi})}-\tau_{y}(\boldsymbol{A})-R(p)
$$

Remark that $\sqrt{J_{2}(.)}$ is the pseudo norm of the tensor deviatoric part implied in the plastic phenomenon (where $J_{2}$ is the second invariant). The initial threshold $\tau_{y}$ depends on the pressure (as it is usual in soil mechanics), on the friction coefficient $\beta$ related to the friction angle $\theta_{f}\left(\beta=\tan \left(\theta_{f}\right)\right)$ and on the cohesion parameter $C_{0}, \tau_{y}(\boldsymbol{A})=C_{0}-\beta \operatorname{tr}(\boldsymbol{A})=\frac{\sigma_{y}}{\sqrt{3}}$. The Drucker - Prager yield criterion is smooth on its deviatoric part and it depends only on two invariants $J_{1}=\operatorname{tr}($.$) and J_{2}$. Moreover, for friction angles below $30^{\circ}$ (Desrues, 2002), it gives a good approximation of the Mohr - Coulomb yield criterion which is classically used for sand. Other models can be used to define the soil plasticity such as those proposed by Vermeer and Nova. Since we use the dual dissipation potential, we get the complementary laws usally obtained from the stress formulation (cf. table I),

$$
\begin{aligned}
& \dot{\boldsymbol{\varepsilon}} \in \partial_{\boldsymbol{\sigma}_{s}^{i r}} \varphi^{*}\left(\boldsymbol{\sigma}_{s}^{i r}, \boldsymbol{A}, \boldsymbol{\chi}, R\right)=\mathbb{R}^{3 \times 3} \\
& \dot{\boldsymbol{\varepsilon}}_{s}^{p}=\dot{\lambda} \frac{\partial F}{\partial \boldsymbol{A}}(\boldsymbol{A}, \boldsymbol{\chi}, R)=\dot{\lambda}\left[\frac{\boldsymbol{A}-\boldsymbol{\chi}}{2 \sqrt{J_{2}(\boldsymbol{A}-\boldsymbol{\chi})}}+\beta \boldsymbol{I}\right] \\
& \dot{\boldsymbol{\alpha}}=\dot{\lambda} \frac{\partial F}{\partial \boldsymbol{\chi}}(\boldsymbol{A}, \boldsymbol{\chi}, R)=-\dot{\lambda} \frac{\boldsymbol{A}-\boldsymbol{\chi}}{2 \sqrt{J_{2}(\boldsymbol{A}-\boldsymbol{\chi})}} \\
& \dot{p}=\dot{\lambda} \frac{\partial F}{\partial R}(\boldsymbol{A}, \boldsymbol{\chi}, R)=-\dot{\lambda},
\end{aligned}
$$

where $\dot{\lambda}$ is the plastic multiplier always nonnegative. Its value can be found with the plastic condition $F=0$ and the consistency condition $\dot{F}=0$,

$$
\left\{\begin{array} { l } 
{ F = 0 } \\
{ \dot { F } = 0 }
\end{array} \Rightarrow \left\{\begin{array}{l}
\sqrt{J_{2}(\boldsymbol{A}-\boldsymbol{\chi})}=\tau_{y}(\boldsymbol{A})+R(p) \\
\dot{\lambda}=\frac{1}{H_{i}+\frac{H_{k}}{2}}\left(\frac{\boldsymbol{A}-\boldsymbol{\chi}}{2\left(\tau_{y}(\boldsymbol{A})+R(p)\right)}+\beta \boldsymbol{I}\right): \dot{\boldsymbol{A}}
\end{array}\right.\right.
$$

In contrast to the $1 \mathrm{D}$ case, we cannot explicitely express a $3 \mathrm{D}$ dissipation potential depending on flow variables.

\subsection{Unilateral wire network model}

According to the 1D model, we neglect the dissipation effects, and we focus on the free energy. Its stiffness cannot be reduced to the stiffness of the wire and has to account for the wire distribution in the sample, assumed to be 
isotropic in the following. Due to the entanglement of the wire network, it is convenient to consider continuously differentiable free energy to derive smooth relations between strain and stress at the macroscopic level. A model directly derived from the isotropic linear elasticity may be expressed in the principal directions; the strain and stress tensors assumed to be coaxial. This property is lost when the unilaterality is considered and we can derive two different models starting either from a strain approach or a stress approach. For instance, using the Lamé coefficients $\lambda_{w}, \mu_{w}$ and the strain principal values denoted $\varepsilon_{w}^{1}, \varepsilon_{w}^{2}, \varepsilon_{w}^{3}$ (we introduce the notations $\widetilde{\varepsilon}_{w}=\operatorname{diag}\left(\varepsilon_{w}^{1}, \varepsilon_{w}^{2}, \varepsilon_{w}^{3}\right)$ and $\left\langle\widetilde{\varepsilon}_{w}\right\rangle=$ $\operatorname{diag}\left(\left\langle\varepsilon_{w}^{1}\right\rangle,\left\langle\varepsilon_{w}^{2}\right\rangle,\left\langle\varepsilon_{w}^{3}\right\rangle\right)$, where $\operatorname{diag}(a, b, c)$ is a diagonal matrix with the diagonal coefficients $a, b$ and $c$ ) the free energy may be defined by,

$$
\psi_{w}\left(\varepsilon_{w}\right)=\frac{\lambda_{w}}{2}\left\langle\varepsilon_{w}^{1}+\varepsilon_{w}^{2}+\varepsilon_{w}^{3}\right\rangle^{2}+\mu_{w}\left(\left\langle\varepsilon_{w}^{1}\right\rangle^{2}+\left\langle\varepsilon_{w}^{2}\right\rangle^{2}+\left\langle\varepsilon_{w}^{3}\right\rangle^{2}\right)
$$

The first term describes the bulk unilateral behavior of the wire network activated by the trace of the strain. The second part concerns the shear component which is not activated in all directions simultaneously but according to the sign of the strain principal values. With a stress approach we could formulate a similar expression for the free enthalpy but using the principal values of the wire stress tensor.

$$
\mathscr{G}_{w}\left(\boldsymbol{\sigma}_{w}\right)=\frac{1-2 \nu_{w}}{6 E_{w}}\left\langle\sigma_{w}^{1}+\sigma_{w}^{2}+\sigma_{w}^{3}\right\rangle^{2}+\frac{1+\nu_{w}}{2 E_{w}}\left(\left\langle S_{w}^{1}\right\rangle^{2}+\left\langle S_{w}^{2}\right\rangle^{2}+\left\langle S_{w}^{3}\right\rangle^{2}\right)
$$

where $\boldsymbol{S}_{w}$ is the deviatoric part of $\boldsymbol{\sigma}_{w}$. The stress expressed in the principal directions is easily derived from (19),

$$
\tilde{\boldsymbol{\sigma}}_{w}^{r}=\frac{\mathrm{d} \psi_{w}}{\mathrm{~d} \widetilde{\boldsymbol{\varepsilon}}_{w}}\left(\widetilde{\boldsymbol{\varepsilon}}_{w}\right)=\lambda_{w}\left\langle\operatorname{tr}\left(\widetilde{\boldsymbol{\varepsilon}}_{w}\right)\right\rangle \boldsymbol{I}+2 \mu_{w}\left\langle\widetilde{\boldsymbol{\varepsilon}}_{w}\right\rangle
$$

In the current frame, the strain-stress relationship has the form,

$$
\boldsymbol{\sigma}_{w}^{r}=\lambda_{w}\left\langle\operatorname{tr}\left(\varepsilon_{w}\right)\right\rangle \boldsymbol{I}+2 \mu_{w} \boldsymbol{P}\left\langle\widetilde{\boldsymbol{\varepsilon}}_{w}\right\rangle \boldsymbol{P}^{T}
$$

where $\boldsymbol{P}$ depending on $\boldsymbol{\varepsilon}_{w}$ is the transformation matrix from the principal directions to the current ones. The expression $\boldsymbol{P}\left\langle\widetilde{\boldsymbol{\varepsilon}}_{w}\right\rangle \boldsymbol{P}^{T}$ is the positive part of the wire strain tensor denoted $\varepsilon_{w}^{z}$. The convexity of the free energy is easily satisfied for $\mu_{w}=0$ because the trace is a linear operator. When $\mu_{w} \neq 0$, the convexity is proved using a composition argument given in (Yang, 1980).

In (De Buhan and Sudret, 1999) a two-phase elastoplastic model for unidirectionally reinforced material is proposed, i.e., the reinforcement network takes the form of a uniaxial stress variable. In our approach, we consider a threedimensional reinforcement coupled with a strong unilateral condition. We use 
the same assumption of equality strain rates $(\S 1.2)$ to superpose both sand and wire models.

\subsection{Models superposition and TexSol potentials}

The previous models are combined according to the 1D approach. Moreover, we introduce two possible initial stresses $\boldsymbol{\sigma}_{w}^{0}$ and $\boldsymbol{\sigma}_{s}^{0}$. These are generated by the deposit process under gravity which may be simulated by a discrete element software (Dubois and Jean, 2003), (Moreau, 1999). Then we can reasonably assume that principal values of $\boldsymbol{\sigma}_{w}^{0}$ are non negatives. We define the corresponding initial strains using the elastic parts of the previous models, $\boldsymbol{\varepsilon}_{w}^{0}=\mathbb{K}_{w}^{-1} \boldsymbol{\sigma}_{w}^{0}$ and $\boldsymbol{\varepsilon}_{s}^{0}=\mathbb{K}_{s}^{-1} \boldsymbol{\sigma}_{s}^{0}$, where $\mathbb{K}_{w}=\lambda_{w} \boldsymbol{I} \otimes \boldsymbol{I}+2 \mu_{w} \mathbb{I}$. The total free energy is then postulated,

$$
\begin{aligned}
\psi\left(\varepsilon, \varepsilon^{p}, \boldsymbol{\alpha}, p\right)= & \frac{1}{2}\left(\varepsilon-\varepsilon^{p}+\varepsilon_{s}^{0}\right): \mathbb{K}_{s}\left(\varepsilon-\varepsilon^{p}+\varepsilon_{s}^{0}\right) \\
& +\frac{\lambda_{w}}{2}\left\langle\operatorname{tr}\left(\varepsilon+\varepsilon_{w}^{0}\right)\right\rangle^{2}+\mu_{w}\left(\varepsilon+\varepsilon_{w}^{0}\right)^{\geq}:\left(\varepsilon+\varepsilon_{w}^{0}\right)^{\geq} \\
& +\frac{H_{k}}{2} \boldsymbol{\alpha}: \boldsymbol{\alpha}+\frac{H_{i}}{2} p^{2} .
\end{aligned}
$$

The state laws are derived,

$$
\begin{aligned}
\boldsymbol{\sigma}^{r} & =\frac{\partial \psi}{\partial \boldsymbol{\varepsilon}}=\mathbb{K}_{s}\left(\boldsymbol{\varepsilon}-\boldsymbol{\varepsilon}^{p}\right)+\boldsymbol{\sigma}_{s}^{0}+\lambda_{w}\left\langle\operatorname{tr}\left(\boldsymbol{\varepsilon}+\boldsymbol{\varepsilon}_{w}^{0}\right)\right\rangle \boldsymbol{I}+2 \mu_{w}\left(\boldsymbol{\varepsilon}+\boldsymbol{\varepsilon}_{w}^{0}\right)^{\geq} \\
\boldsymbol{A} & =-\frac{\partial \psi}{\partial \boldsymbol{\varepsilon}^{p}}=\mathbb{K}_{s}\left(\varepsilon-\boldsymbol{\varepsilon}^{p}\right)+\boldsymbol{\sigma}_{s}^{0} \\
\boldsymbol{\chi} & =-\frac{\partial \psi}{\partial \boldsymbol{\alpha}}=-H_{k} \boldsymbol{\alpha} \\
R & =-\frac{\partial \psi}{\partial p}=-H_{i} p
\end{aligned}
$$

The complementary laws are derived considering the dual dissipation potential of the sand alone (cf. equation (15)). In the simple case where $\mu_{w}=0$, we can complete the dual stress formulation by computing the Legendre Fenchel transformation of the free energy $\psi$ (denoted in this case $\psi_{\circ}$ ) via the proposition 1.

$$
\psi_{\circ}^{*}\left(\boldsymbol{\sigma}^{r}, \boldsymbol{\varepsilon}^{p}, \boldsymbol{\alpha}, p\right)=\left\{\begin{array}{c}
\frac{1}{2}\left(\boldsymbol{\sigma}^{r}+\mathbb{K}_{s}\left(\boldsymbol{\varepsilon}^{p}-\boldsymbol{\varepsilon}_{s}^{0}\right)+\boldsymbol{\sigma}_{w}^{0}\right):\left(\mathbb{K}_{s}+\mathbb{K}_{w}^{\circ}\right)^{-1}\left(\boldsymbol{\sigma}^{r}+\mathbb{K}_{s}\left(\boldsymbol{\varepsilon}^{p}-\boldsymbol{\varepsilon}_{s}^{0}\right)+\boldsymbol{\sigma}_{w}^{0}\right) \\
-\frac{1}{2}\left(\left(\boldsymbol{\varepsilon}^{p}-\boldsymbol{\varepsilon}_{s}^{0}\right): \mathbb{K}_{s}\left(\boldsymbol{\varepsilon}^{p}-\boldsymbol{\varepsilon}_{s}^{0}\right)+H_{k} \boldsymbol{\alpha}: \boldsymbol{\alpha}+H_{i} p^{2}\right)-\frac{1}{2} \boldsymbol{\varepsilon}_{w}^{0}: \boldsymbol{\sigma}_{w}^{0} \\
\text { if } \operatorname{tr}\left(\boldsymbol{\sigma}^{r}+\mathbb{K}_{s}\left(\boldsymbol{\varepsilon}^{p}-\boldsymbol{\varepsilon}_{s}^{0}-\boldsymbol{\varepsilon}_{w}^{0}\right)\right) \geq 0 \\
\frac{1}{2}\left(\boldsymbol{\sigma}^{r}+\mathbb{K}_{s}\left(\boldsymbol{\varepsilon}^{p}-\boldsymbol{\varepsilon}_{s}^{0}\right)\right): \mathbb{K}_{s}^{-1}\left(\boldsymbol{\sigma}^{r}+\mathbb{K}_{s}\left(\boldsymbol{\varepsilon}^{p}-\boldsymbol{\varepsilon}_{s}^{0}\right)\right) \\
-\frac{1}{2}\left(\left(\boldsymbol{\varepsilon}^{p}-\boldsymbol{\varepsilon}_{s}^{0}\right): \mathbb{K}_{s}\left(\boldsymbol{\varepsilon}^{p}-\boldsymbol{\varepsilon}_{s}^{0}\right)+H_{k} \boldsymbol{\alpha}: \boldsymbol{\alpha}+H_{i} p^{2}\right) \\
\text { if } \operatorname{tr}\left(\boldsymbol{\sigma}^{r}+\mathbb{K}_{s}\left(\boldsymbol{\varepsilon}^{p}-\boldsymbol{\varepsilon}_{s}^{0}-\boldsymbol{\varepsilon}_{w}^{0}\right)\right) \leq 0,
\end{array}\right.
$$


where $\mathbb{K}_{w}^{\circ}=\lambda_{w} \boldsymbol{I} \otimes \boldsymbol{I}$. In this expression it is difficult to distinguish a contribution of the unilaterality of the wire network as given in (20). Consequently the two approaches (19) and (20) are no more equivalent when unilaterality occurs (Lucchesi and al., 2000). The Legendre - Fenchel transformation cannot be catched in the more general case.

\section{Numerical development}

Starting from a consistent thermodynamic model for the TexSol, the next step consists in implementing it in a finite element software (Keryvin, 1999), (Kichenin and Charras, 2003). We discuss then responses provided by the simulation of simple compression / traction tests according to the expected behaviors detailed in section 1 .

\subsection{Numerical implementation}

The variables being known at step $n-1$, we have to compute them at step $n$ using a predicted value of the strain increment $\Delta \varepsilon_{n}$. For the sake of simplicity, the initial stresses are neglected $\left(\boldsymbol{\sigma}_{s}^{0}=\boldsymbol{\sigma}_{w}^{0}=\mathbf{0}\right)$. Two sets of variables, $\left(\boldsymbol{\sigma}_{s, n}, \boldsymbol{\chi}_{n}, p_{n}\right)$ for the sand and $\left(\boldsymbol{\sigma}_{w, n}\right)$ for wire network are computed simultaneously. The stress in the wire network is directly deduced from the potential defined by (19). For sand, the relations given in (14), (17) and (18) can be reduced to three equations depending on the three unknowns $\left(\boldsymbol{\sigma}_{s, n}, \boldsymbol{\chi}_{n}, p_{n}\right)$. This system is solved by the Newton - Raphson method applied to the following residuals $\boldsymbol{Q}_{n}^{\alpha} ; \alpha=1,2,3$.

$$
\begin{aligned}
\boldsymbol{Q}_{n}^{1}= & \frac{p_{n}-p_{n-1}}{2\left(R_{n}+\tau_{y, n}\right)}\left(\boldsymbol{S}_{s, n}-\boldsymbol{\chi}_{n}+2\left(R_{n}+\tau_{y, n}\right) \beta \boldsymbol{I}\right)+\Delta \boldsymbol{\varepsilon}_{n} \\
& -\mathbb{K}_{s}^{-1}\left(\boldsymbol{\sigma}_{s, n}-\boldsymbol{\sigma}_{s, n-1}\right) \\
\boldsymbol{Q}_{n}^{2}= & \frac{H_{k}\left(p_{n}-p_{n-1}\right)}{2\left(R_{n}+\tau_{y, n}\right)}\left(\boldsymbol{S}_{s, n}-\boldsymbol{\chi}_{n}\right)+\boldsymbol{\chi}_{n}-\boldsymbol{\chi}_{n-1} \\
Q_{n}^{3}= & \frac{1}{\left(R_{n}+\tau_{y, n}\right)\left(H_{k}+2 H_{i}\right)}\left(\boldsymbol{S}_{s, n}-\boldsymbol{\chi}_{n}+2\left(R_{n}+\tau_{y, n}\right) \beta \boldsymbol{I}\right):\left(\boldsymbol{\sigma}_{s, n}-\boldsymbol{\sigma}_{s, n-1}\right) \\
& +p_{n}-p_{n-1}
\end{aligned}
$$

where $\alpha=1$ corresponds to equations (2), (14) $)_{1},(17)_{2,4}$ and $(18)_{1}, \alpha=2$ corresponds to equations $(14)_{3},(17)_{3,4}$ and (18) $)_{1}$ and finally $\alpha=3$ corresponds to equations $(17)_{4}$ and $(18)_{1,2}$ (in all these equations, $R_{n}$ is calculated using the equation $\left.(14)_{4}\right)$. Classically, the Taylor development is defined as follows

$$
\boldsymbol{Q}_{n, i+1}^{\alpha}=\boldsymbol{Q}_{n, i}^{\alpha}+\left(\frac{\partial \boldsymbol{Q}_{n}^{\alpha}}{\partial \boldsymbol{\sigma}_{s, n}}\right)_{i} \delta \boldsymbol{\sigma}_{s, n, i+1}+\left(\frac{\partial \boldsymbol{Q}_{n}^{\alpha}}{\partial \boldsymbol{\chi}_{n}}\right)_{i} \delta \boldsymbol{\chi}_{n, i+1}+\left(\frac{\partial \boldsymbol{Q}_{n}^{\alpha}}{\partial p_{n}}\right)_{i} \delta p_{n, i+1}
$$


The analytical formulations of the local tangent matrix coefficients are

$$
\begin{aligned}
\frac{\partial \boldsymbol{Q}_{n}^{1}}{\partial \boldsymbol{\sigma}_{s, n}}= & \frac{p_{n}-p_{n-1}}{2\left(R_{n}+\tau_{y, n}\right)^{2}}\left[\left(R_{n}+\tau_{y, n}\right) \mathbb{D}+\beta\left(\boldsymbol{S}_{s, n}-\boldsymbol{\chi}_{n}\right) \otimes \boldsymbol{I}\right]-\mathbb{K}_{s}{ }^{-1} \\
\frac{\partial \boldsymbol{Q}_{n}^{1}}{\partial \boldsymbol{\chi}_{n}}= & -\frac{p_{n}-p_{n-1}}{2\left(R_{n}+\tau_{y, n}\right)} \mathbb{I} \\
\frac{\partial \boldsymbol{Q}_{n}^{1}}{\partial p_{n}}= & \frac{R_{n}+\tau_{y, n}+H_{i}\left(p_{n}-p_{n-1}\right)}{2\left(R_{n}+\tau_{y, n}\right)^{2}}\left(\boldsymbol{S}_{s, n}-\boldsymbol{\chi}_{n}\right)+\beta \boldsymbol{I} \\
\frac{\partial \boldsymbol{Q}_{n}^{2}}{\partial \boldsymbol{\sigma}_{s, n}}= & \frac{H_{k}\left(p_{n}-p_{n-1}\right)}{2\left(R_{n}+\tau_{y, n}\right)^{2}}\left[\left(R_{n}+\tau_{y, n}\right) \mathbb{D}+\beta\left(\boldsymbol{S}_{s, n}-\boldsymbol{\chi}_{n}\right) \otimes \boldsymbol{I}\right] \\
\frac{\partial \boldsymbol{Q}_{n}^{2}}{\partial \boldsymbol{\chi}_{n}}= & \left(1-H_{k} \frac{p_{n}-p_{n-1}}{2\left(R_{n}+\tau_{y, n}\right)}\right) \mathbb{I} \\
\frac{\partial \boldsymbol{Q}_{n}^{2}}{\partial p_{n}}= & H_{k} \frac{R_{n}+\tau_{y, n}+H_{i}\left(p_{n}-p_{n-1}\right)}{2\left(R_{n}+\tau_{y, n}\right)^{2}}\left(\boldsymbol{S}_{s, n}-\boldsymbol{\chi}_{n}\right) \\
\frac{\partial Q_{n}^{3}}{\partial \boldsymbol{\sigma}_{s, n}}= & C_{n}^{1}\left(2 \boldsymbol{S}_{s, n}-\boldsymbol{S}_{s, n-1}-\boldsymbol{\chi}_{n}+2\left(R_{n}+\tau_{y, n}\right) \beta \boldsymbol{I}\right) \\
& +C_{n}^{2}\left(\boldsymbol{S}_{s, n}-\boldsymbol{\chi}_{n}\right):\left(\boldsymbol{\sigma}_{s, n}-\boldsymbol{\sigma}_{s, n-1}\right) \boldsymbol{I} \\
\frac{\partial Q_{n}^{3}}{\partial \boldsymbol{\chi}_{n}}= & -C_{n}^{1}\left(\boldsymbol{\sigma}_{s, n}-\boldsymbol{\sigma}_{s, n-1}\right) \\
\frac{\partial Q_{n}^{3}}{\partial p_{n}}= & 1+C_{n}^{3}\left(\boldsymbol{S}_{s, n}-\boldsymbol{\chi}_{n}-2\left(R_{n}+\tau_{y, n}\right) \beta \boldsymbol{I}\right):\left(\boldsymbol{\sigma}_{s, n}-\boldsymbol{\sigma}_{s, n-1}\right),
\end{aligned}
$$

where $C_{n}^{1}\left(R_{n}, \tau_{y, n}\right)=\frac{1}{\left(R_{n}+\tau_{y, n}\right)\left(H_{k}+2 H_{i}\right)}, C_{n}^{2}\left(R_{n}, \tau_{y, n}\right)=\frac{\beta C_{n}^{1}}{R_{n}+\tau_{y, n}}$ and finally $C_{n}^{3}\left(R_{n}, \tau_{y, n}\right)=\frac{H_{i} C_{n}^{1}}{R_{n}+\tau_{y, n}}$. The algorithm is schematized in the table II (where $\left.\left(\zeta_{n}^{1}, \zeta_{n}^{2}, \zeta_{n}^{3}\right)=\left(\boldsymbol{\sigma}_{s, n}, \boldsymbol{\chi}_{n}, p_{n}\right)\right)$. This last one being quite complex for sand, we have compared the results given by the previous integration law and strategy with the one developped in the Cast3M software where a Drucker - Prager model is available. Since we got a good agreement with both implementations, we focus our attention on the coupled sand/wire model of TexSol involving a unilateral behavior. A consistent tangent matrix can be used to increase the convergence rate (Simo and Hugues, 1998).

\subsection{Patch test}

In a first step, the simple patch test considered is a single Q1-Lagrange hexahedron finite element submitted to a traction/compression loading (cf. figure 6). More precisely, a confinement pressure is prescribed via a cohesion behavior on the material (Radjaï and al., 2001) depending on a single coefficient $C_{0}$. A displacement is imposed on the upperside. Four models are compared to emphasize the relevance of the two unilateral behavior laws. Two of them are considered to obtain some limit behaviors; the first one denoted Sand, is free of wire; the second one denoted Reinforced sand, is a superposition of a sand model and an elastic "bilateral" model of the reinforcement. The "unilateral" TexSol model introduced in $§ 3.3$ is denoted Texsol. A particular model is added denoted Spherical Texsol corresponding to the previous one 


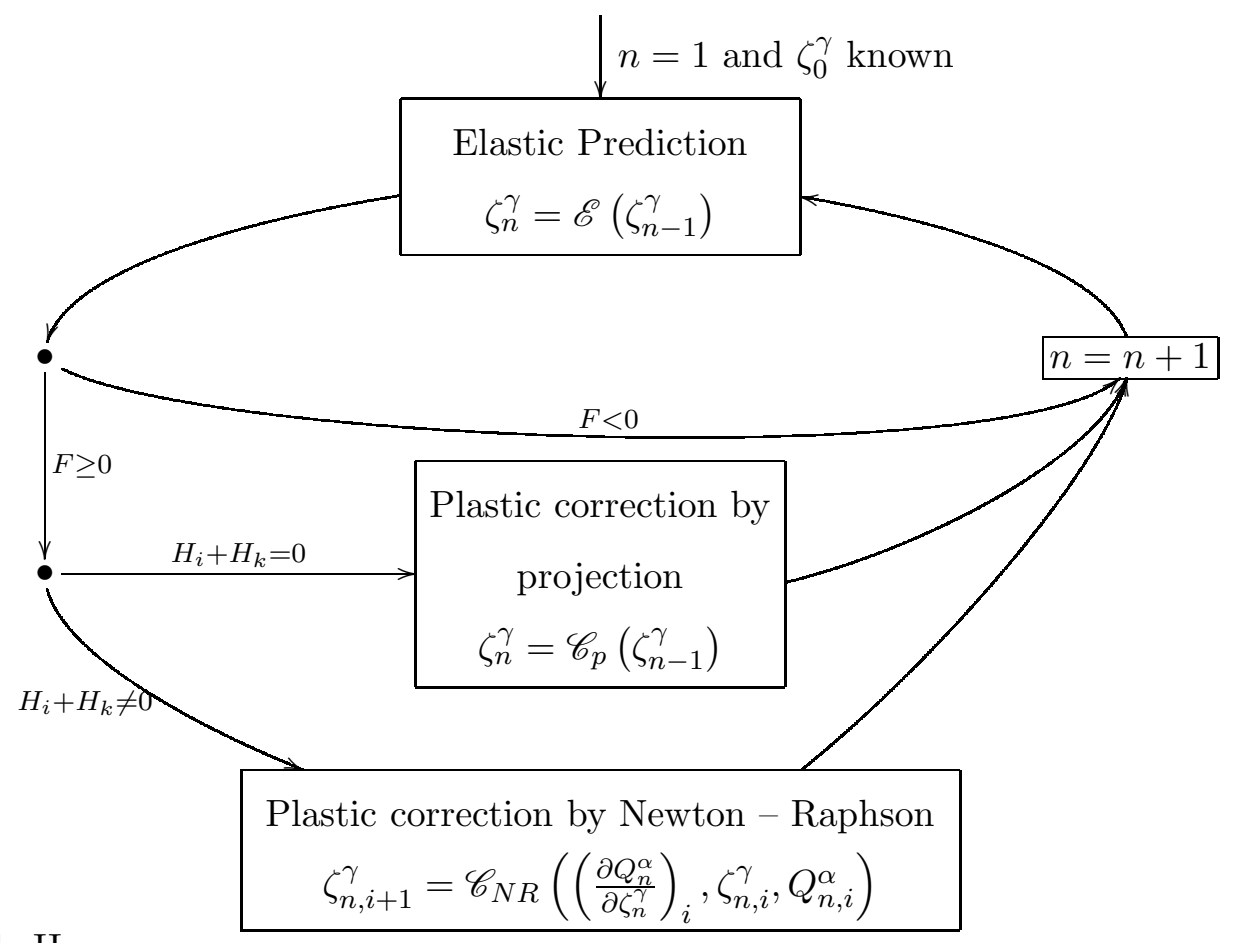

Table II

Solution algorithm

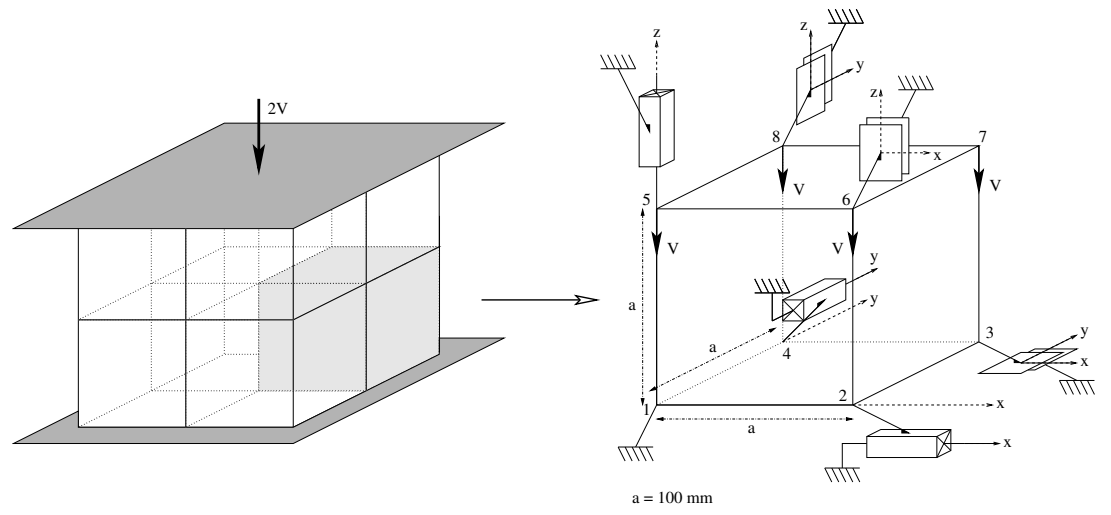

Fig. 6. Patch test

with $\mu_{w}=0$.

- Elasticity: $E_{s}=200 \mathrm{MPa}, \nu_{s}=0.4, E_{w}=100 \mathrm{MPa}, \nu_{w}=0.3$

- Plasticity: $C_{0}=50 \mathrm{kPa}, \theta_{f}=0.1, H_{k}=1 \mathrm{MPa}, H_{i}=1 \mathrm{MPa}$

Sand and Reinforced sand appear clearly as two elastic bounds for TexSol models (cf. figure 7). At this stage, Spherical Texsol does not differ from Sand. On the contrary, the Texsol is close to Sand in compression and close to Reinforced sand in traction. For the two loadings the limit models appear to be the upper bounds. For a loading-unloading traction process, the Texsol model behaves almost like Reinforced sand as expected (cf. figure 8). Spherical 


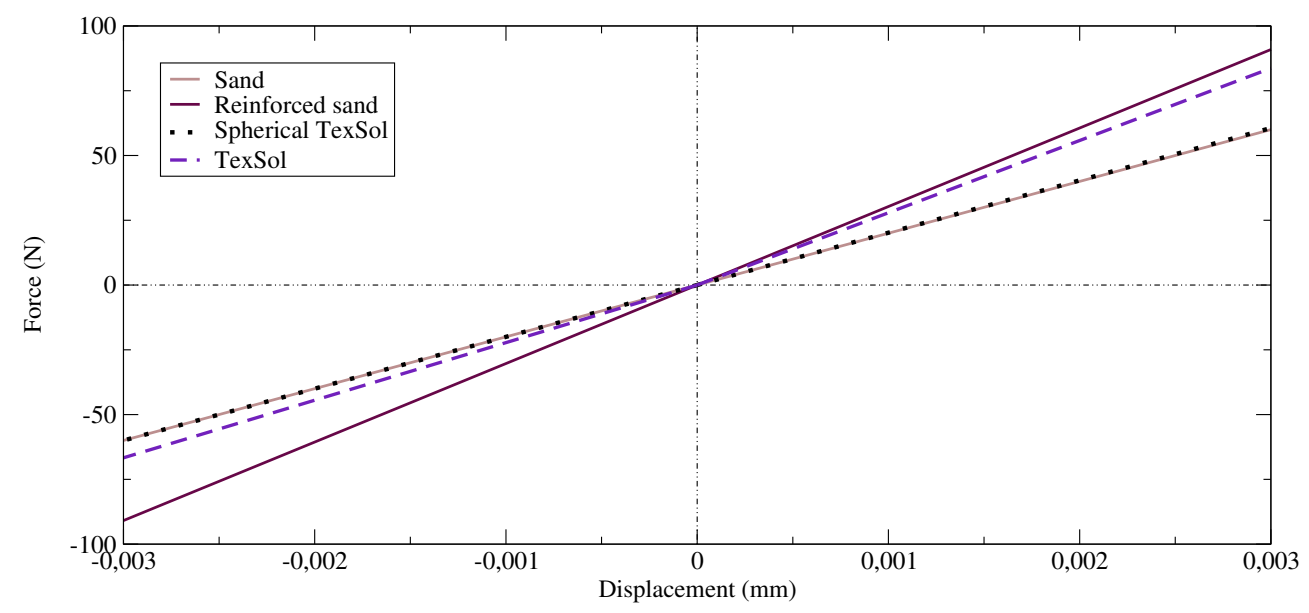

Fig. 7. Zoom on the elastic range of the models

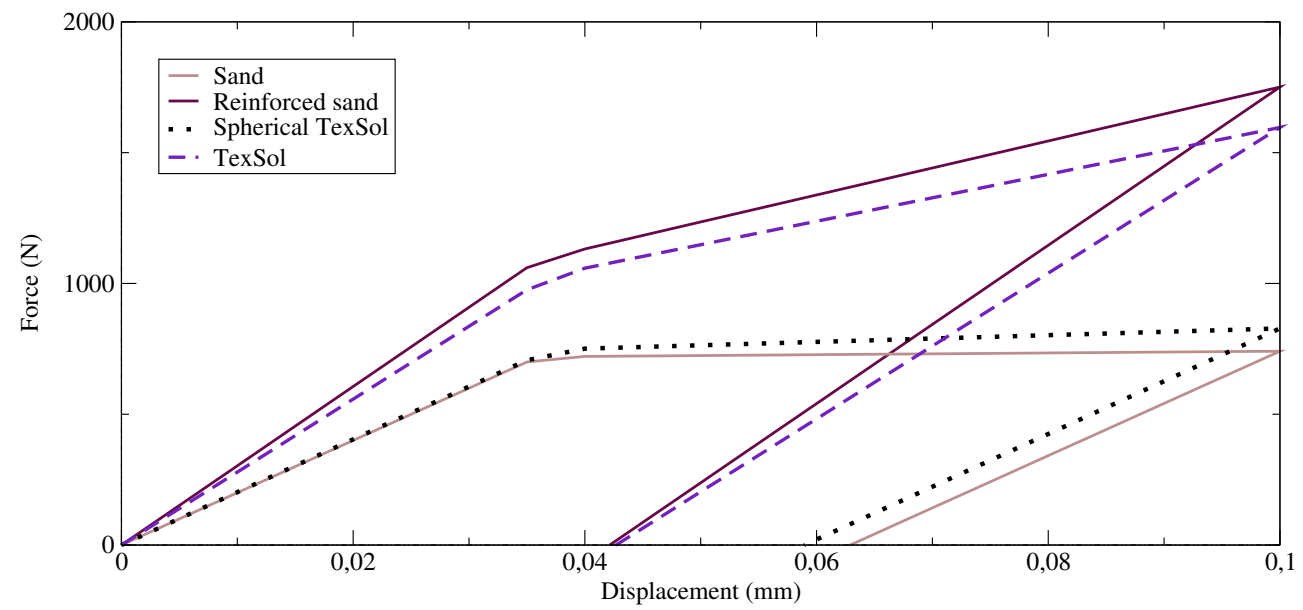

Fig. 8. Different material behaviors on a traction patch test

Texsol does not improve significantly Sand (cf. figure 8 and 9). Consequently, Spherical Texsol does not account for the numerical results given in figure 3 for the same kind of experiment - even roughly.

\subsection{Cyclic loading}

TexSol embankments may be submitted to vibrational solicitations. A cyclic test based on the test represented in figure 10 (where the displacements are fixed on the lower side and the sollicitation controlled by force) is performed to highlight the contribution of the "unilateral" reinforcement due to the wire network. The friction angle is changed to apply a greater amplitude of loading on it: $\theta_{f}=0.02$. Reinforced sand, Texsol and Sand cyclic behaviors are 


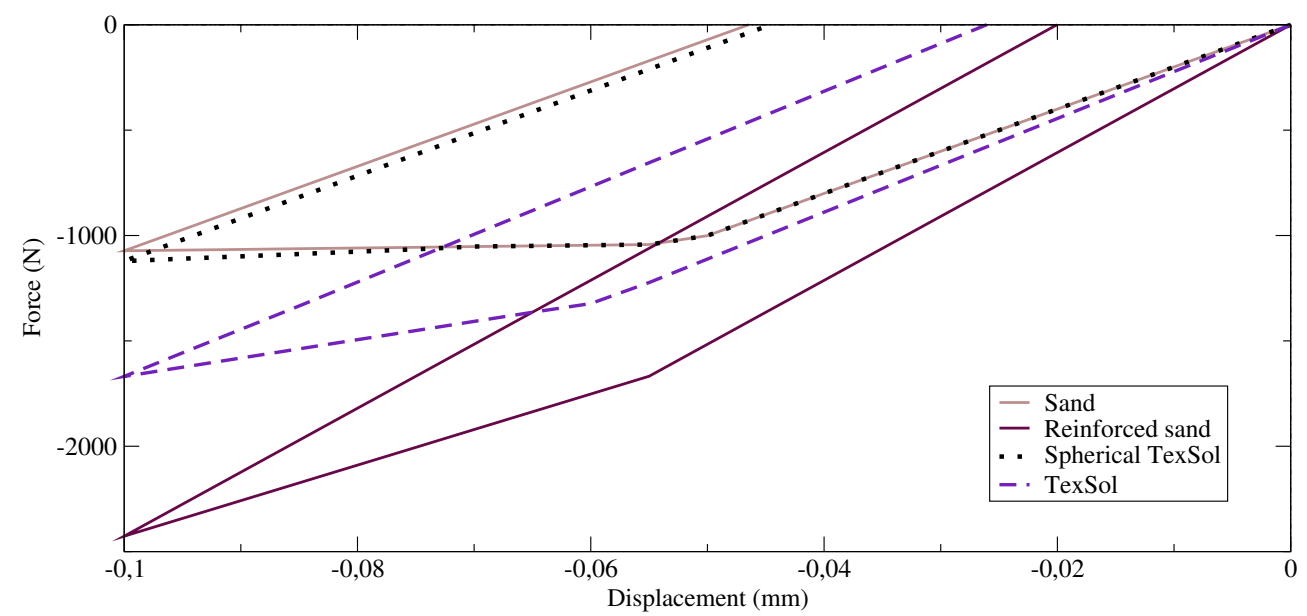

Fig. 9. Different material behaviors on a compression patch test

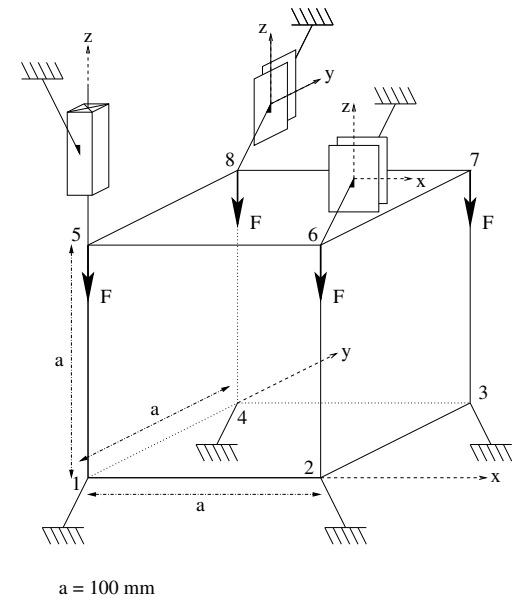

Fig. 10. Force managed traction / compression test

compared in figure 11. For the three models, the response tends to be stabilized after 20 cycles. But for Reinforced sand and Texsol the stabilization is reached before 10 cycles. Moreover, the residual displacement of Texsol is close to that of Reinforced sand and five times smaller than that of Sand. This last result highlights the advantages of TexSol reinforcement. Another effect of the "unilateral" wire in the Texsol model is clearly illustrated by the curvature changes when the displacement switches sign from positive to negative.

\subsection{Compression test}

In soil mechanics, it is usual to carry out a triaxial test with a prescribed confinement pressure (cf. fig 12). Considering the previous numerical results of $\S 4.2$, the Spherical Texsol model is no more studied. Only the three other cases 


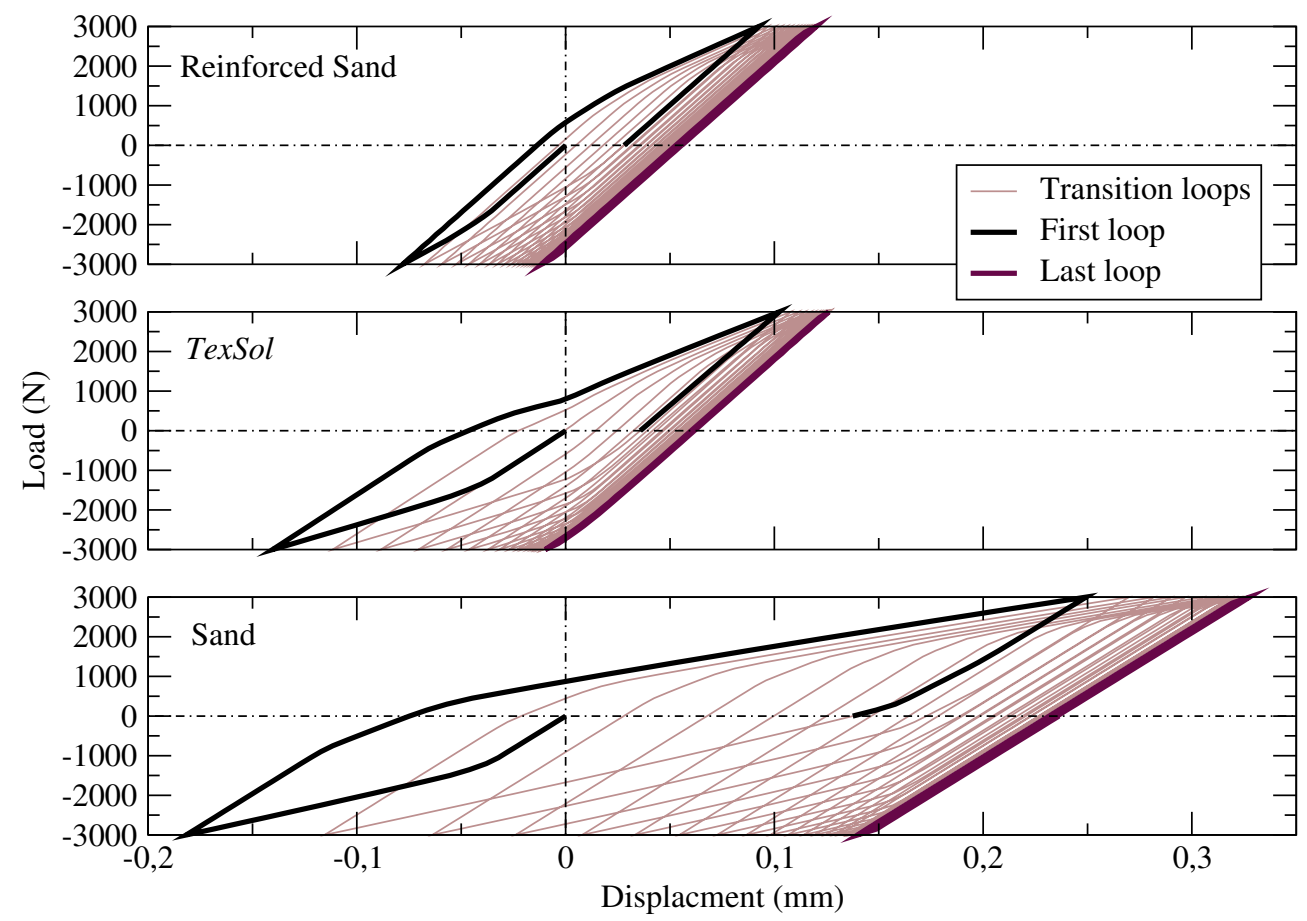

Fig. 11. Reinforced sand, TexSol and Sand behaviors on a cyclic test (20 compression traction load loops)

are compared in a compression test $(0.1 \mathrm{~mm}$ of vertical displacement computed in 100 iterations). The mesh is composed of 400 elements as described in (Laniel, 2004). We consider Q1-Lagrange hexahedron finite elements except for the central part which is meshed with R1-Lagrange pentahedron finite elements. The contribution of the wire in TexSol to the strength is illustrated
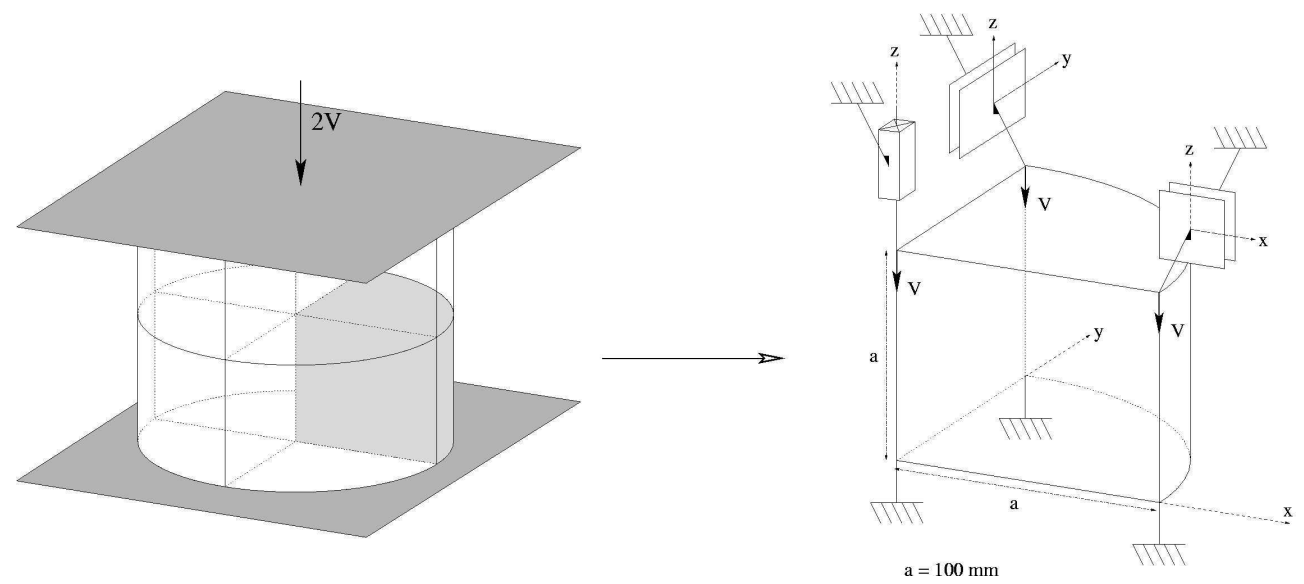

Fig. 12. Compression test

by the spatial distribution of two stresses: the full stress $\boldsymbol{\sigma}$ and the wire stress $\boldsymbol{\sigma}_{w}$. The distribution of the full stress is identical in the three models with a level for Texsol which lies between the two others. The main part of stress is located at the center of the bulk except a localized concentration at the right 


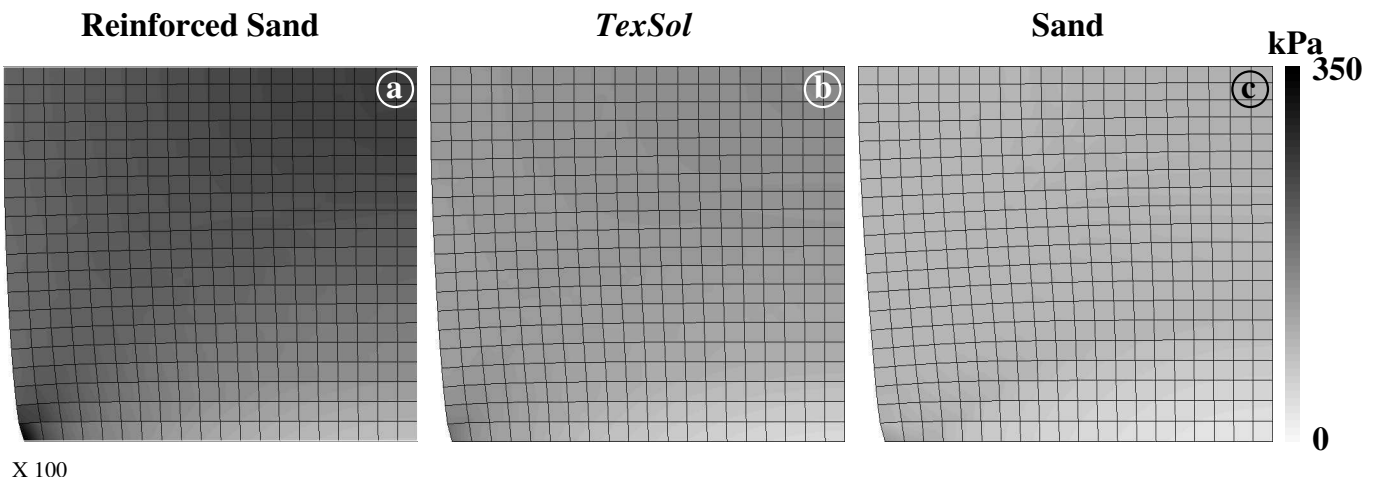

Fig. 13. Deviatoric stress

lower corner. The contribution of the wire to the stress tensor $\left(\boldsymbol{\sigma}_{w}\right)$ is split into its deviatoric and spherical (pressure) part. Both parts are identically zero for Sand (cf. figure 14c and 15c). The elasticity of the reinforcement is activated

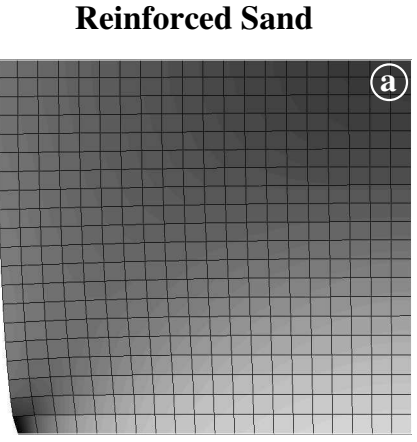

X 100
TexSol

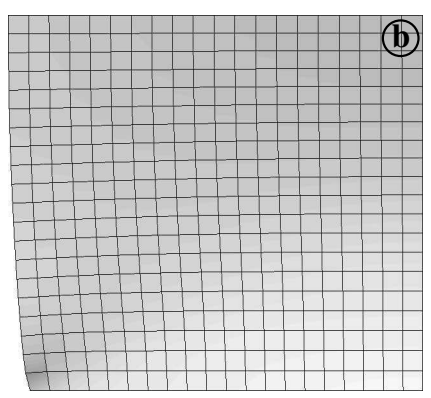

Sand

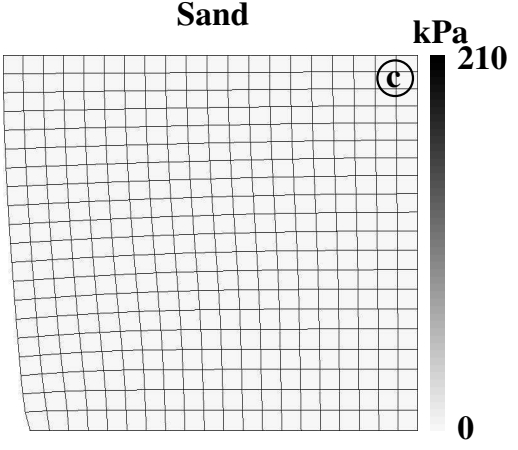

Fig. 14. Wire deviatoric stress

only in tensile directions for Texsol and in all directions for Reinforced sand; this explains the difference in the levels of deviatoric stress for the total stress (figure 13) and for the wire stress (figure 14). The nature of the reinforcement

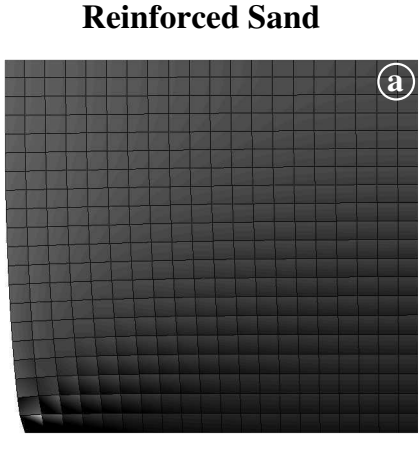

X 100
TexSol

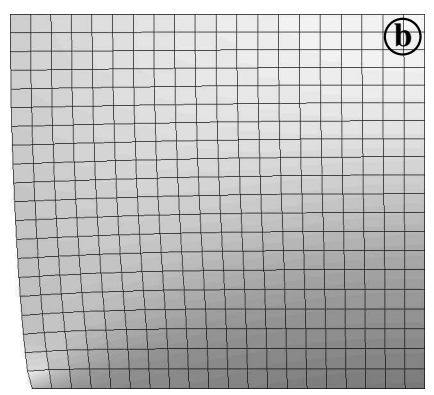

Fig. 15. Wire pressure

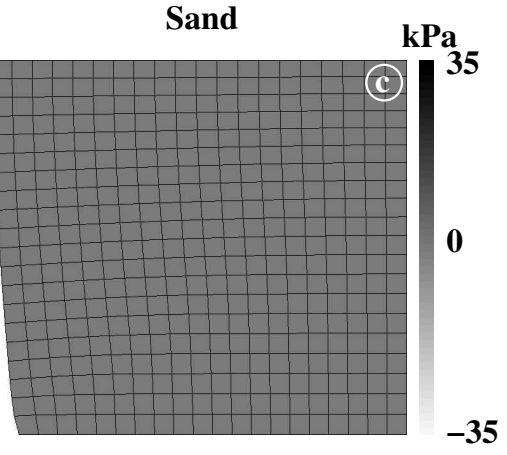

due to wire is clearly illustrated in figure 15 . Obviously, the wire pressure in the Sand sample vanishes. It is negative in the Texsol wire (traction behavior) 
according to the unilaterality condition expressed in equation (21) whereas the pressure in the reinforcement of Reinforced sand is almost everywhere positive.

\section{Conclusion and perspectives}

In this paper a consistent thermodynamic model was proposed to account for numerical experiments (because of the absence of exploitable real experiments on TexSol). The key-point was the introduction of a "unilateral" elasticity which models the wire network. An elastic plastic model was superposed on the previous model to obtain both strain and stress formulations when it was possible. Using a finite element method, we validated qualitatively the expected behavior.

The main perspective of this work is the identification of the mechanical parameters of the two-component model by numerical experiments which are currently underway. In a more general framework, it is useful to employ an orthotropic model for the wire network.

\section{Acknowledgement}

Thanks to Dr. Keryvin from the LARMAUR (Rennes) for his theoric and logistics supports. 


\section{References}

Cambou, B., Jean, M., 2001: Micromécanique des matériaux granulaires. Hermès. Science-Paris.

Clarke, F.H., 1983: Optimization and nonsmooth analysis. Wiley-Interscience Philadelphia. Republished as Clarke, F.H., 1990, Classics in Applied Mathematics, vol. 5, SIAM, New-York.

De Buhan, P., Sudret, B., 1999: A two-phase elastoplastic model for unidirectionally reinforced materials. Eur. Jour. Mech. A/Sol. 18, p. 995-1012, 1999.

Desrues, J., 2002: Limitations du choix de l'angle de frottement pour le critère de plasticité de Drucker - Prager. Rev. Fra. Gen. Civ. vol. 6, n 1, p. 853-862.

Drucker, D., Prager, W., 1952: Soil mechanics and plastic analysis of limit design. Quart. Appl. Math., vol. 10, p. 157-165.

Dubois, F., Jean, M., 2003: LMGC90 une plateforme de développement dédiée à la modélisation des problèmes d'interaction. $6^{\text {th }}$ CNCS Giens, vol. 1, p. 111-118.

Fremond, M., 2002: Non-Smooth Thermo-mechanics. Springer-Verlag Berlin Heidelberg New York.

Halphen, B., Nguyen, QS., 1975: Sur les matériaux standards généralisés. Journal de Mécanique, $\mathrm{n}^{\circ}$ 14, p. 39-63.

Jean, M., 1999: The non smooth contact dynamics method. Computer Methods in Applied Mechanic and Engineering 177 (Special issue), p. 235-257.

Keryvin, V., 1999: Contribution à la modélisation de l'endommagement localisé. PhD Thesis, Université de Poitier, LMPM/LMA.

Khay, M., Gigan, J-P., 1990: TEXSOL - Ouvrage de soutènement. LCPC.

Kichenin, J., Charras, T., 2003: CAST3M - Implantation d'une nouvelle loi d'évolution / loi de comportement mécanique. SEMT/LM2S, 2003.

Laniel, R., 2004: Simulation des procédés d'indentation et de rayage par éléments finis et éléments distincts. DEA, Université de Rennes I \& INSA.

Laniel, R., Mouraille, O., Pagano, S., Dubois, F., Alart, P., 2005: Numerical modelling of reinforced geomaterials by wires using the Non Smooth Contact Dynamics. $4^{\text {th }}$ CMIS Hannover.

Leflaive, E.,Khay, M., Blivet, J-C., 1985: Un nouveau matériaux: le TEXSOL. Travaux, $\mathrm{n}^{\circ}$ 602, p. 1-3.

Lemaitre, J., Chaboche, J-L., 1990: Mechanics of solid materials. Cambridge.

Lucchesi, M., Pandovani, C., Pasquinelli, G., 2000: Thermodynamics of notension materials. Int. Jour. Sol. Struct 37, p. 6581-6604.

Moreau, J-J., 1966: Fonctionnelles convexes. Séminaire Equations aux dérivés partielle, Collège de France.

Moreau, J-J., 1999: Numerical aspects of the sweeping process. Comput. Methods Appl. Mech. Engrg., n 177, p. 329-349.

Mouraille, O., 2004: Etude sur le comportement d'un matériau longueur interne: le TexSol. DEA, Université de Montpellier II. 
Radjaï, F., Preechawuttipong, I., Peyroux, R., 2001: Cohesive granular texture. in (Vermeer and al., 2001), p. 149-162.

Simo, J-C., Hugues, T.J.R, 1998: Computational inelasticity. Springer-Verlag.

Suquet, P., 1982: Plasticité et homogénéisation. PhD Thesis, Université Pierre et Marie Curie, 1982.

Vermeer, P.A., Diebels, S., Ehlers, W., Hermann, H-J., Ludwig, S., Ramm, E., (Eds.), 2001: Continuous and discontinuous modelling of cohesive frictional materials. Springer Berlin.

Villard, P., 1988: Etude du renforcement des sables par des fils continus. PhD Thesis, Université de Nantes, ENSM.

Villard, P., Jouve, P., 1989: Behavior of granular materials reinforced by continuous threads. Computers and Geothechnics, vol. 7, $\mathrm{n}^{\circ} 1$ and 2, p. 83-98. Wood, D.M., 1990: Soil behaviour and critical state soil mechanics. Cambridge. Yang, WH., 1980: A generalized Von Mises criterion for yield and fracture. J. Applied Mechanics n 47, p. 297-300. 